\title{
Clemens Berger
}

\section{Un groupoïde simplicial comme modèle de} l'espace des chemins

\author{
Bulletin de la S. M. F., tome 123, no 1 (1995), p. 1-32 \\ <http://www.numdam.org/item?id=BSMF_1995_123_1_1_0>
}

(C) Bulletin de la S. M. F., 1995, tous droits réservés.

L'accès aux archives de la revue «Bulletin de la S. M. F. » (http: //smf.emath.fr/Publications/Bulletin/Presentation.html) implique l'accord avec les conditions générales d'utilisation (http://www.numdam.org/ conditions). Toute utilisation commerciale ou impression systématique est constitutive d'une infraction pénale. Toute copie ou impression de ce fichier doit contenir la présente mention de copyright.

\section{Numdam}


Bull. Soc. math. France,

123, 1995, p. 1-32.

\title{
UN GROUPOÏDE SIMPLICIAL COMME MODÈLE DE L'ESPACE DES CHEMINS
}

\author{
PAR \\ Clemens BERGER $(*)$
}

\begin{abstract}
RÉSUMÉ. - Pour tout ensemble simplicial nous définissons un groupoïde simplicial qui sert de modèle de l'espace des chemins au sens de Quillen. Le fibré en lacets associé fournit une interprétation géométrique du fibré universel de Kan et de la construction cobar cubique de Baues. Nous obtenons en particulier une description algébrique des groupes d'homotopie d'un ensemble simplicial pointé qui étend la présentabilité du groupe de Poincaré aux groupes d'homotopie supérieurs. Les représentants des classes d'homotopie peuvent être donnés sous forme algébrique ou simpliciale.

Abstract. - For each simplicial set we define a simplicial groupoid which serves as path-object in Quillen's sense. The associated loop bundle gives a geometric interpretation of Kan's universal bundle and of Baues' cubical cobar construction. In particular we obtain an algebraic description of the homotopy groups of a pointed simplicial set which extends the presentability of the Poincare group to the higher dimensional homotopy groups. The representatives of the homotopy classes may be given in algebraic or simplicial terms.
\end{abstract}

\section{Introduction}

Selon Quillen [Q], un modèle simplicial de l'espace des chemins est essentiellement la donnée d'un graphe simplicial $e_{X}, s_{X}: I X \rightrightarrows X$ tel que la projection sur les extrémités $\left(e_{X}, s_{X}\right): I X \rightarrow X \times X$ soit une fibration. Si l'ensemble simplicial $X$ vérifie la condition d'extension de Kan, l'objet fonctionnel $X^{\Delta[1]}$ a les propriétés requises, ce qui règle la question d'existence, au moins du point de vue de l'homotopie.

Pour faire valoir l'aspect combinatoire du concept de chemin, nous présentons ici un foncteur $I$ qui sert de modèle de l'espace des chemins

(*) Texte reçu le 12 janvier 1993.

C. Berger, Université de Nice-Sophia Antipolis, Laboratoire J.A. Dieudonné, URA du CNRS no 162, Parc Valrose, F-06108 Nice Cedex 2.

Email : cberger@math.unice.fr.

Classification AMS : 18 G 30, 55 J 10, 55 E 05, 55 D 35, 57 F 30. 
pour la catégorie entière des ensembles simpliciaux. Le foncteur associe à l'ensemble simplicial $X$ un groupoïde simplicial $I X$ libre en toute dimension et contenant $X$ comme ensemble d'unités. L'axiome de Quillen découle aisément de cette structure algébrique. La construction reste pourtant compatible avec la réalisation géométrique en ce sens qu'il existe une application de graphes topologiques $|I X| \rightarrow|X|^{[0,1]}$ (à valeurs dans l'espace des chemins de $|X|)$ qui préserve à homotopie près la structure groupoïdale.

Comme tout modèle de l'espace des chemins, le groupoïde simplicial $I X$ induit à la fois une relation d'homotopie (appelée prismatique) et un modèle combinatoire du fibré en lacets (défini pour tout ensemble simplicial pointé et connexe $X$ ).

L'homotopie prismatique est eo ipso une relation d'équivalence, vaguement comparable à la clôture transitive de l'homotopie cylindrique. Elle offre avant tout un formalisme commode pour exprimer des homotopies «à longue distance combinatoire».

L'application principale de la construction $I X$ concerne cependant les isomorphismes de connexion du fibré en lacets, noté $\Omega X \hookrightarrow P X \rightarrow X$. La double nature algébrico-prismatique de la fibre $\Omega X$ permet en effet non seulement de définir les groupes d'homotopie de $\Omega X$ de manière quasiment algébrique, mais encore d'associer à tout représentant algébrique d'une classe d'homotopie $[\omega] \in \pi_{n}(\Omega X)$, un représentant simplicial canonique

$$
\omega_{\mathrm{sph}}: \Sigma^{n+1}(\omega) \longrightarrow X
$$

de la classe d'homotopie adjointe $\left[\left|\omega_{\mathrm{sph}}\right|\right] \in \pi_{n+1}(|X|)$. Cette forme explicite de l'isomorphisme de connexion

$$
\partial_{n+1}(X): \pi_{n+1}(|X|) \stackrel{\sim}{\longrightarrow} \pi_{n}(\Omega X)
$$

redonne pour $n=0$ la présentation du groupe de Poincaré de $X$ par générateurs et relations. En dimension supérieure, elle semble nouvelle.

L'idée d'une définition algébrique des groupes d'homotopie (supérieurs) remonte à KAN [K1] qui utilisait à cet effet les isomorphismes de connexion du fibré universel $G X \hookrightarrow E X \rightarrow X$. Grâce à une inclusion naturelle de groupes simpliciaux $G X \hookrightarrow \Omega X$, la construction du représentant simplicial adjoint s'applique également aux représentants algébriques de Kan. Il est remarquable dans ce contexte que l'espace total du fibré universel de Kan admette une contraction prismatique.

L'autre modèle de l'espace de lacets que nous avons trouvé dans la littérature est le modèle cubique $\Omega_{B} X$ de Baues, directement calqué sur

TOME $123-1995-\mathrm{N}^{\circ} 1$ 
la construction cobar d'Adams [Bau]. Ce modèle $\Omega_{B} X$, subdivisé simplicialement, est également plongeable dans $\Omega X$. Le plongement revient à rendre «prismatique» l'approximation par un $n$-cube de l'espace des chemins reliant deux sommets (fixes) du $(n+1)$-simplexe affine.

L'exposé sera subdivisé en trois parties (comparer avec [Be]) :

- la première introduit le groupoïde simplicial et étudie ses propriétés locales;

- la seconde partie traite le fibré en lacets;

- la troisième partie établit les liens avec le fibré universel de Kan et la construction cobar d'Adams-Baues.

Je voudrais remercier F. SERGERAERT pour les encouragements répétés qui ont accompagné la rédaction de ce texte. Sa vision «effective» de la structure simpliciale fut le support indispensable des idées développées ici.

\section{Le groupoïde simplicial des prismes}

En ce qui concerne la catégorie des ensembles simpliciaux, nous suivons de près les notations et terminologie habituelles (cf. [Cu], [G-Z]). Compte tenu de l'application spécifique que nous ferons des graphes nous avons préféré modifier légèrement la terminologie «standard», comme c'est indiqué ci-dessous.

\subsection{Le groupoïde librement engendré par un graphe.}

1.1.a. - Un graphe $\Gamma$ sera présenté sous forme d'un quadruplet $(X, \Xi, e, s)$, où $X$ désigne l'ensemble des sommets, $\Xi$ l'ensemble des arêtes et $(e, s): \Xi \rightarrow X \times X$ la fonction entrée-sortie. Pour deux sommets $x, y \in X$, on notera $\Gamma_{x, y}$ l'ensemble des arêtes d'entrée $x$ et de sortie $y$.

Un $\Gamma$-trajet d'entrée $x$ et de sortie $y$ est un mot formel $\gamma=\xi_{1}^{\varepsilon_{1}} \xi_{2}^{\varepsilon_{2}} \cdots \xi_{\ell}^{\varepsilon_{\ell}}$ de l'alphabet $\Xi^{ \pm 1}$ vérifiant $x=e\left(\xi_{1}^{\varepsilon_{1}}\right), s\left(\xi_{k}^{\varepsilon_{k}}\right)=e\left(\xi_{k+1}^{\varepsilon_{k+1}}\right), 1 \leq k<\ell$, $s\left(\xi_{\ell}^{\varepsilon_{\ell}}\right)=y$, où par convention

$$
(e, s)\left(\xi^{\varepsilon}\right)= \begin{cases}(e, s)(\xi) & \text { si } \varepsilon=1 \\ (s, e)(\xi) & \text { si } \varepsilon=-1\end{cases}
$$

Si l'entrée et la sortie coïncident, on admet en plus un $\Gamma$-trajet trivial, noté $1_{x}$, avec $x \in X$.

1.1.b. - Un $\Gamma$-trajet est réduit s'il ne contient pas d'aller retour $\xi^{\varepsilon} \xi^{-\varepsilon}$. Il existe une opération de réduction de $\Gamma$-trajets associant à tout $\Gamma$-trajet $\gamma$ l'unique $\Gamma$-trajet réduit $\gamma_{\text {red }}$ qui suit $\gamma$ en «évitant» les aller retour. 
1.1.c. - Le graphe $\Gamma=(X, \Xi, e, s)$ est plongé dans un graphe $F \Gamma=$ $(X, F \Xi, e, s)$ dont l'ensemble d'arêtes $F \Xi$ est l'ensemble des $\Gamma$-trajets réduits. La composition

$$
\begin{aligned}
(F \Gamma)_{x, y} \times(F \Gamma)_{y, z} & \longrightarrow(F \Gamma)_{x, z} \\
\left(\gamma_{1}, \gamma_{2}\right) & \longmapsto\left(\gamma_{1} \gamma_{2}\right)_{\mathrm{red}}
\end{aligned}
$$

munit $F \Gamma$ d'une structure de groupoïde.

Le groupoïde $F \Gamma$ est en fait librement engendré par $\Gamma$, puisqu'il est caractérisé par la propriété universelle que tout morphisme de graphes $\varphi: \Gamma \rightarrow G$ à valeurs dans un groupoïde $G$ s'étend de manière unique en un morphisme de groupoïdes $\Phi: F \Gamma \rightarrow G$.

Pour plus de détails, nous renvoyons à $[\mathrm{D}]$.

1.2. Définitions. - Soit $X$ un ensemble simplicial.

Un $n$-prisme élémentaire de $X$ est un couple $[\xi, i]$ composé d'un $(n+1)$ simplexe $\xi \in X_{n+1}$ et d'un indice directionnel $i$, avec $0 \leq i \leq n$, tels que $\xi$ n'appartienne pas à l'image de l'opérateur de dégénérescence $s_{i}: X_{n} \rightarrow X_{n+1}$.

L'ensemble $\Xi_{n}$ des $n$-prismes élémentaires de $X$ forme l'ensemble des arêtes du graphe $\Gamma_{n}(X)=\left(X_{n}, \Xi_{n}, e, s\right)$, où les fonctions entrée et sortie sont définies par

$$
\begin{aligned}
(e, s): \Xi_{n} & \longrightarrow X_{n} \times X_{n} \\
{[\xi, i] } & \longmapsto\left(\partial_{i+1} \xi, \partial_{i} \xi\right) .
\end{aligned}
$$

Un $n$-prisme de $X$ est alors un $\Gamma_{n}(X)$-trajet réduit.

Le groupoïde libre des $n$-prismes de $X$ sera noté $(I X)_{n}$. Le groupoïde simplicial des prismes de $X$ est la réunion disjointe $I X$ des $(I X)_{n}, n \geq 0$, qu'on munit d'une structure simpliciale qui prolonge celle de l'ensemble d'unités $\left\{1_{x} \mid x \in X\right\}$.

Les opérateurs de face $\partial_{j}:(I X)_{n} \rightarrow(I X)_{n-1}$ (resp. de dégénérescence $\left.s_{j}:(I X)_{n} \rightarrow(I X)_{n+1}\right)$ sont en fait définis par extension canonique des opérateurs graphiques suivants $\left([\xi, i] \in \Xi_{n}\right)$ :

$$
\left\{\begin{aligned}
\partial_{j}[\xi, i]= \begin{cases}{\left[\partial_{j} \xi, i-1\right]} & \text { si } j<i, \\
1_{\partial_{i} \partial_{i+1} \xi} & \text { si } j=i, \\
{\left[\partial_{j+1} \xi, i\right]} & \text { si } j>i\end{cases} \\
s_{j}[\xi, i]= \begin{cases}{\left[s_{j} \xi, i+1\right]} & \text { si } j<i, \\
{\left[s_{i} \xi, i+1\right]\left[s_{i+1} \xi, i\right]} & \text { si } j=i, \\
{\left[s_{j+1} \xi, i\right]} & \text { si } j>i .\end{cases}
\end{aligned}\right.
$$

TOME $123-1995-\mathrm{N}^{\circ} 1$ 
Si $\partial_{j} \xi=s_{i-1} x\left(\right.$ resp. $\left.\partial_{j+1} \xi=s_{i} x\right)$, on identifie $\left[\partial_{j} \xi, i-1\right]$ (resp. $\left.\left[\partial_{j+1} \xi, i\right]\right)$ au prisme trivial $1_{x}$.

Les morphismes structurels du groupoïde simplicial $I X$ seront notés

$$
\begin{aligned}
&\left(e_{X}, s_{X}\right): I X \longrightarrow X \times X: p \longmapsto\left(e_{X}(p), s_{X}(p)\right), \\
& i_{X}: X \longrightarrow I X: x \longmapsto 1_{x} .
\end{aligned}
$$

Deux morphismes $f, g: Y \rightarrow X$ sont prismatiquement homotopes (relativement au sous-ensemble simplicial $Z$ de $Y$ ) s'il existe un morphisme $H: Y \rightarrow I X$ tel que $e_{X} H=f$ et $s_{X} H=g\left(\right.$ et $\left.i_{X} \circ f_{\mid Z}=H_{\mid Z}=i_{X} \circ g_{\mid Z}\right)$. On notera également $H: f \sim g$ (rel. $Z$ ).

1.2.b. - Il est souvent utile de réaliser le groupoïde simplicial $I X$ comme quotient de l'ensemble simplicial $\widehat{I} X$. A cet effet, nous plongeons le graphe $\Gamma_{n}(X)$ dans le graphe $\widehat{\Gamma}_{n}(X)=\left(X_{n}, \widehat{\Xi}_{n}, e, s\right)$, où

$$
\widehat{\Xi}_{n}=\Xi_{n} \cup\left\{\left[s_{i} x, i\right] ; x \in X_{n}, 0 \leq i \leq n\right\} .
$$

On définit alors la catégorie involutive libre $(\widehat{I} X)_{n}$ des $\widehat{\Gamma}_{n}(X)$-trajets (y compris les non réduits). Les formules 1.2.a appliquées sans aucune identification définissent des opérateurs de face $\hat{\partial}_{j}:(\widehat{I} X)_{n} \rightarrow(\widehat{I} X)_{n-1}$ et de dégénérescence $\hat{s}_{j}:(\widehat{I} X)_{n} \rightarrow(\widehat{I} X)_{n+1}$ qui vérifient les identités simpliciales habituelles. Le groupoïde simplicial $I X$ s'obtient alors à partir de $\widehat{I} X$ par un passage au quotient

$$
q: \widehat{I} X \longrightarrow I X: p \longmapsto p_{\text {red }},
$$

où la réduction se fait en deux étapes :

- le $\widehat{\Gamma}_{n}(X)$-trajet $p \in(\widehat{I} X)_{n}$ est d'abord transformé en $\Gamma_{n}(X)$-trajet en supprimant toutes les composantes appartenant à $\left(\widehat{\Xi}_{n} \backslash \Xi_{n}\right)^{ \pm 1}$;

- le $\Gamma_{n}(X)$-trajet résultant est ensuite transformé en $n$-prisme $p_{\text {red }}$ appartenant à $(I X)_{n}$ selon le procédé de réduction habituel 1.1.b.

Tout $n$-prisme $p \in(I X)_{n}$ est en particulier un $\widehat{\Gamma}_{n}(X)$-trajet, ce qui permet de récupérer les opérateurs simpliciaux de $I X$ par les formules

$$
\partial_{j} p=\left(\hat{\partial}_{j} p\right)_{\mathrm{red}} \quad \text { et } \quad s_{j} p=\left(\hat{s}_{j} p\right)_{\mathrm{red}}=\hat{s}_{j} p, \quad 0 \leq j \leq n .
$$

Enfin, les constructions $\widehat{I}$ et $I$ s'étendent en foncteurs : pour tout morphisme $f: X \rightarrow Y$ on définit le morphisme $\widehat{I} f: \widehat{I} X \rightarrow \widehat{I} Y$ par

$$
\left[\xi_{1}, i_{1}\right]^{\varepsilon_{1}}\left[\xi_{2}, i_{2}\right]^{\varepsilon_{2}} \cdots\left[\xi_{\ell}, i_{\ell}\right]^{\varepsilon_{\ell}} \longmapsto\left[f \xi_{1}, i_{1}\right]^{\varepsilon_{1}}\left[f \xi_{2}, i_{2}\right]^{\varepsilon_{2}} \cdots\left[f \xi_{\ell}, i_{\ell}\right]^{\varepsilon_{\ell}} .
$$

Le morphisme If en est déduit par passage au quotient. 
1.3. Proposition.

(a) Pour tout ensemble simplicial $X$, le morphisme entrée-sortie $\left(e_{X}, s_{X}\right): I X \rightarrow X \times X$ est une fibration de Kan.

(b) La relation d'homotopie prismatique est une relation d'équivalence.

(c) Soient $f, g: Y \rightarrow X$ deux morphismes prismatiquement homotopes rel. $Z$. Les réalisations géométriques $|f|$ et $|g|$ sont alors homotopes rel. $|Z|$.

Preuve.

(a) Il faut montrer que pour tout couple de simplexes $(x, y)$ dans $X_{n} \times X_{n}, n \geq 0$, la fibre $(I X)_{x, y}=\left(e_{X}, s_{X}\right)^{-1}((x),(y))$ vérifie la condition d'extension de Kan. Grâce à la structure de groupoïde de $I X$, l'ensemble simplicial $(I X)_{x, y}$ est muni d'une opération de Mal'cev partielle

$$
\begin{aligned}
M:(I X)_{x, y} \times(I X)_{x, y} \times(I X)_{x, y} & \longrightarrow(I X)_{x, y} \\
(p, q, r) & \longmapsto p q^{-1} r
\end{aligned}
$$

Les identités de Mal'cev $M(p, p, r)=r$ et $M(p, r, r)=p$ impliquent alors que tout problème d'extension de Kan admet une solution dans $(I X)_{x, y}$. Nous renvoyons pour cela à la démonstration bien connue du fait qu'un groupe simplicial vérifie la condition de Kan, car celle-ci n'utilise que la structure de Mal'cev du groupe (cf. [Cu, 3.1]).

(b) Réflexivité, symétrie et transitivité de l'homotopie prismatique sont les conséquences de l'existence d'unités, d'inverses et du composé de deux prismes adjacents.

(c) L'énoncé constitue la jonction entre la combinatoire des prismes et la topologie des chemins. Sa preuve demande un effort technique considérable et sera faite au $\S 1.8$. La difficulté principale provient des identifications introduites par le passage au quotient $\widehat{I} X \rightarrow I X$. Nous signalons dans ce contexte que la construction $\widehat{I} X$ vérifie bien 1.3 .b, mais en général pas l'axiome de Quillen 1.3.a.

1.4. Remarque. - Si l'ensemble simplicial $X$ vérifie la condition de Kan, alors une homotopie prismatique $p: x \sim y$ (rel. le bord) entre simplexes de $X$ peut toujours être réalisé par une homotopie élémentaire $[\xi, i]: x \sim y$ (rel. le bord), le choix de l'indice directionnel $i$ étant libre. Pour $i=\operatorname{dim} x$, on retrouve ainsi la relation d'homotopie initialement proposée par KAN [K1]. C'est cette propriété «localisante» de la condition de Kan qui permet de travailler avec l'homotopie cylindrique $Y \times \Delta[1] \rightarrow X$ dès que $X$ vérifie la condition de Kan. L'homotopie prismatique $Y \rightarrow I X$ fournit par contre une relation d'homotopie qui reflète dans une large mesure la situation topologique, même si $X$ n'est pas de Kan. 
Les $§ \S 1.5-1.6$ préparent à la Proposition 1.7. Il s'agit de «paramétrer » tout $n$-prisme par une $(n+1)$-boule simplicialement subdivisée de sorte que les opérateurs de face formels soient induits par des opérateurs de bord géométriques. Les technicités qui suivent sont malheureusement incontournables étant donnée l'importance de la Proposition 1.7 pour l'étude du fibré en lacets.

\section{5. $N$-éléments et $N$-sphères.}

On dira que l'ensemble simplicial $Y_{1}$ est une $C W$-subdivision de l'ensemble simplicial $Y_{2}$ si le $C W$-complexe $\left|Y_{1}\right|$ est une subdivision cellulaire du $C W$-complexe $\left|Y_{2}\right|$, i.e. s'il existe un homéomorphisme $\left|Y_{1}\right| \stackrel{\sim}{\longrightarrow}\left|Y_{2}\right|$ tel que l'image de toute cellule de $\left|Y_{1}\right|$ soit incluse dans une cellule de $\left|Y_{2}\right|$. La relation d'équivalence engendrée par la relation de $C W$-subdivision sera appelée $C W$-équivalence. Une $C W$-équivalence $\left|Y_{1}\right| \stackrel{\sim}{\longrightarrow}\left|Y_{2}\right|$ est donc le composé d'un nombre fini d'homéomorphismes qui sont soit des $C W$-subdivisions soit des inverses de $C W$-subdivisions.

Un $n$-élément simplicial $E^{n}$ est un ensemble simplicial $C W$-équivalent au $n$-élément standard $\Delta[n]$. Le réalisé $\left|E^{n}\right|$ hérite en particulier d'une structure canonique de $n$-variété linéaire par morceaux. Le bord $b E^{n}$ de $E^{n}$ est défini comme l'unique sous-ensemble simplicial de $E^{n}$ dont le réalisé s'identifie au bord topologique de $\left|E^{n}\right|$.

Une $n$-sphère simpliciale $\Sigma^{n}$ est un ensemble simplicial $C W$-équivalent à la $n$-sphère standard $S^{n}=\Delta[n] / b \Delta[n]$. Nous signalons qu'une $C W$ équivalence $\left|\Sigma^{n}\right| \stackrel{\sim}{\longrightarrow}\left|S^{n}\right|$ est forcément une $C W$-subdivision; $\Sigma^{n}$ est donc munie d'un point base canonique correspondant à l'unique 0-cellule de $\left|S^{n}\right|$.

Le théorème de Newman (cf. [R-S, 3.1]) reste valable dans le contexte semi-simplicial : pour tout $n$-élément simplicial $E^{n}$ inclus dans une $n$ sphère simpliciale $\Sigma^{n}$, l'adhérence du complémentaire $\left(\Sigma^{n} \backslash E^{n}\right) \cup b E^{n}$ est également un n-élément simplicial.

\subsection{Domaine et domaine $\partial$-réduit d'un prisme.}

Soit $X$ un ensemble simplicial. Un $\widehat{\Gamma}_{n}(X)$-trajet de longueur un, $[\xi, i]^{\varepsilon} \in(\widehat{I} X)_{n}$, définit le diagramme

$$
\Delta[n] \underset{s(i, \varepsilon)}{\stackrel{e(i, \varepsilon)}{\longrightarrow}} \Delta[n+1] \stackrel{\xi}{\longrightarrow} X,
$$

dans lequel les morphismes $\xi, \xi \circ e(i, \varepsilon)$ et $\xi \circ s(i, \varepsilon)$ sont les représentants canoniques des simplexes $\xi \in X_{n+1}$ et $e_{X}\left([\xi, i]^{\varepsilon}\right), s_{X}\left([\xi, i]^{\varepsilon}\right) \in X_{n}$.

Deux diagrammes $\Delta[n] \underset{s_{i}}{\stackrel{e_{i}}{\longrightarrow}} Y \stackrel{\xi_{i}}{\longrightarrow} X$, pour $i=1,2$, tels que 
$s_{1}(\Delta[n])=e_{2}(\Delta[n])$ sont amalgamés dans le diagramme

$$
\Delta[n] \stackrel{e_{1}}{\underset{s_{2}}{\longrightarrow}} Y_{1} \square Y_{2} \stackrel{\xi_{1} \square \xi_{2}}{\longrightarrow} X,
$$

dans lequel l'ensemble simplicial $Y_{1} \square Y_{2}$ est défini par

$$
Y_{1} \square Y_{2}=\left(Y_{1} \dot{\cup} Y_{2}\right) /\left(s_{1}(\Delta[n]) \sim e_{2}(\Delta[n])\right) .
$$

1.6.a. - Un $\widehat{\Gamma}_{n}(X)$-trajet $p=\left[\xi_{1}, i_{1}\right]^{\varepsilon_{1}} \cdots\left[\xi_{\ell}, i_{\ell}\right]^{\varepsilon_{\ell}} \in(\widehat{I} X)_{n}$ définit donc par amalgamation itérée le diagramme

$$
\Delta[n] \stackrel{e_{1}\left(i_{1}, \varepsilon_{1}\right)}{\underset{s_{\ell}\left(i_{\ell}, \varepsilon_{\ell}\right)}{\longrightarrow}} \prod_{i=1}^{\ell} \Delta[n+1] \stackrel{\stackrel{\ell}{\square} \xi_{k}}{\longrightarrow} X,
$$

dont le morphisme droit sera désigné par $p_{\text {simp }}: \operatorname{Dom}(p) \rightarrow X$. L'ensemble simplicial source $\operatorname{Dom}(p)$ sera appelé le domaine $d u \widehat{\Gamma}_{n}(X)$-trajet $p$. Il est muni d'un $n$-prisme fondamental $\alpha=\left[\alpha_{1}, i_{1}\right]^{\varepsilon_{1}} \cdots\left[\alpha_{\ell}, i_{\ell}\right]^{\varepsilon_{\ell}} \in(\widehat{I} \operatorname{Dom}(p))_{n}$ tel que $\left(\widehat{I} p_{\text {simp }}\right)(\alpha)=p$, en particulier $p_{\text {simp }}\left(\alpha_{k}\right)=\xi_{k}$ pour $1 \leq k \leq \ell$.

Le domaine d'un $\widehat{\Gamma}_{n}(X)$-trajet non trivial $p \in(\widehat{I} X)_{n}$ est un $(n+1)$ élément simplicial dont le bord est composé d'une réunion cosimpliciale de faces latérales (correspondant aux faces du $\widehat{\Gamma}_{n}(X)$-trajet) et de deux faces extrémales (correspondant à l'entrée et la sortie du trajet).

Plus précisément, il existe pour toute suite $0 \leq j_{1}<j_{2}<\cdots<j_{r} \leq n$ d'indices, une inclusion canonique

$$
\widehat{\varepsilon}_{j_{r}} \circ \cdots \circ \widehat{\varepsilon}_{j_{2}} \circ \widehat{\varepsilon}_{j_{1}}: \operatorname{Dom}\left(\hat{\partial}_{j_{1}} \cdots \hat{\partial}_{j_{r}} p\right) \longleftrightarrow \operatorname{Dom}(p)
$$

telle qu'on ait

$$
p_{\text {simp }} \circ \widehat{\varepsilon}_{j_{r}} \circ \cdots \circ \widehat{\varepsilon}_{j_{1}}=\left(\hat{\partial}_{j_{1}} \cdots \hat{\partial}_{j_{r}} p\right)_{\text {simp }}
$$

les inclusions $\widehat{\varepsilon}_{j}$ étant définies en recollant comme ci-dessus des inclusions élémentaires $\operatorname{Dom}\left(\hat{\partial}_{j}[\xi, i]^{\varepsilon}\right) \hookrightarrow \operatorname{Dom}\left([\xi, i]^{\varepsilon}\right)$ déduites de 1.2.a.

1.6.b. - La construction du domaine d'un $\widehat{\Gamma}_{n}(X)$-trajet s'applique en particulier aux $n$-prismes, mais pour un $n$-prisme, le domaine naturel n'est pas l'objet géométrique adéquat, les opérateurs $\partial_{j}$ étant différents des opérateurs $\hat{\partial}_{j}$. Pour en tenir compte, nous allons définir des relations sur l'ensemble simplicial $\operatorname{Dom}(p)$ qui reflètent géométriquement la réduction du $\widehat{\Gamma}_{n}(X)$-trajet $p$ au $n$-prisme associé $p_{\text {red }}$ (cf. 1.2.b). Soit

$$
p=\left[\xi_{1}, i_{1}\right]^{\varepsilon_{1}} \cdots\left[\xi_{\ell}, i_{\ell}\right]^{\varepsilon_{\ell}} \in(\widehat{I} X)_{n} .
$$

TOME $123-1995-\mathrm{N}^{\circ} 1$ 
Correspondant à la première étape de réduction, on définit l'ensemble de relations

$$
R_{p}^{(1)}=\left\{\left(\alpha_{k} \sim s_{i_{k}} \partial_{i_{k}} \alpha_{k}\right) ; \xi_{k}=s_{i_{k}} \partial_{i_{k}} \xi_{k}, 1 \leq k \leq \ell\right\} .
$$

Correspondant à la deuxième étape de réduction, on définit l'ensemble de relations

$$
R_{p}^{(2)}=\left\{\left(\alpha_{k_{1}} \sim \alpha_{k_{2}}\right) ;\left[\xi_{k_{1}}, i_{k_{1}}\right]^{\varepsilon_{k_{1}}}\left[\xi_{k_{2}}, i_{k_{2}}\right]^{\varepsilon_{k_{2}}} \in \mathcal{A}_{p \rightarrow p_{\mathrm{red}}}\right\}
$$

où $\mathcal{A}_{p \rightarrow p_{\text {red }}}$ est l'ensemble des aller retour supprimés lors d'une réduction effective de $p$ vers $p_{\text {red }}$. L'ensemble $\mathcal{A}_{p \rightarrow p_{\text {red }}}$ peut différer selon le schéma de réduction choisi : le mot $a a^{-1} b^{-1} b a$ se réduit par exemple soit par $\left(a a^{-1}\right)\left(b^{-1} b\right) a$ soit par $a\left(a^{-1}\left(b^{-1} b\right) a\right)$. Cela étant, il existe des stratégies de réduction qui la rendent unique. Nous supposons en avoir choisi une.

1.6.c. - Le domaine $\partial$-réduit d'un $n$-prisme non trivial $p \in(I X)_{n}$ est défini comme l'ensemble simplicial quotient

$$
\overline{\operatorname{Dom}}(p)=\operatorname{Dom}(p) /\left(R_{\partial p}^{(1)} \cup R_{\partial p}^{(2)}\right)
$$

où les ensembles de relations de bord $R_{\partial p}^{(1)}$ et $R_{\partial p}^{(2)}$ sont donnés par

$$
\begin{aligned}
R_{\partial p}^{(1)} & =\bigcup_{1 \leq r \leq n} \bigcup_{0 \leq j_{1}<\cdots<j_{r} \leq n} \widehat{\varepsilon}_{j_{r}} \circ \cdots \circ \widehat{\varepsilon}_{j_{1}}\left(R_{\hat{\partial}_{j_{1}} \cdots \hat{\partial}_{j_{r}} p}^{(1)}\right), \\
R_{\partial p}^{(2)} & =\bigcup_{0 \leq j \leq n} \widehat{\varepsilon}_{j}\left(R_{\hat{\partial}_{j} p}^{(2)}\right) .
\end{aligned}
$$

Il résulte des définitions que le morphisme $p_{\text {simp }}$ se factorise par le domaine $\partial$-réduit :

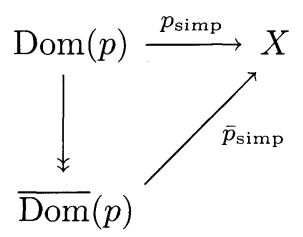

1.7. Proposition. - Soit un n-prisme non trivial $p \in(I X)_{n}$. Le domaine d-réduit $\overline{\operatorname{Dom}}(p)$ est alors un $(n+1)$-élément simplicial muni des faces latérales $\varepsilon_{j}: \operatorname{Dom}\left(\partial_{j} p\right) \rightarrow \overline{\operatorname{Dom}}(p), 0 \leq j \leq n$, et des faces extrémales e, $s: \Delta[n] \hookrightarrow \overline{\operatorname{Dom}}(p)$ telles que:

(a) $\left\{\begin{array}{l}\bar{p}_{\text {simp }} \circ \varepsilon_{j}=\left(\partial_{j} p\right)_{\text {simp }}, \quad 0 \leq j \leq n, \\ \bar{p}_{\text {simp }} \circ e=e_{X}(p), \quad \bar{p}_{\text {simp }} \circ s=s_{X}(p) ;\end{array}\right.$

(b) $b \overline{\operatorname{Dom}}(p)=e(\Delta[n]) \cup \bigcup_{0 \leq j \leq n} \varepsilon_{j}\left(\operatorname{Dom}\left(\partial_{j} p\right)\right) \cup s(\Delta[n])$. 
Preuve. - Il faut d'abord montrer que le passage au quotient $\operatorname{Dom}(p) \rightarrow \overline{\operatorname{Dom}}(p)$ n'affecte pas le «type combinatoire»du $(n+1)$ élément simplicial $\operatorname{Dom}(p)$. Pour cela, les relations de bord $R_{\partial p}^{(1)} \cup R_{\partial p}^{(2)}$ seront introduites une par une selon le schéma suivant : on commence par les «compressions» $\left(\sigma \sim s_{i} \partial_{i} \sigma\right) \in R_{\partial p}^{(1)}$ qu'on traite par ordre de dimension décroissante (en évitant les répétitions). Le simplexe $\sigma$ est alors «standard» au moment de la compression, i.e. il engendre une copie de $\Delta[m]$, où $m=\operatorname{dim} \sigma \leq n$.

Or, d'après un lemme de Barratt [F-P, 3.1.1], on sait que pour tout $m$-simplexe du bord de $\Delta[n+1]$ et pour tout indice $i$ tel que $0 \leq i \leq m$, il existe un homéomorphisme

$$
|\Delta[n+1]| \stackrel{\sim}{\longrightarrow}\left|\Delta[n+1] /\left(\sigma \sim s_{i} \partial_{i} \sigma\right)\right|
$$

qui est une $C W$-subdivision. Par ailleurs, on sait construire pour tout couple $\left(E, \sigma_{E}\right)$ constitué d'un $(n+1)$-élément $E$ et d'un $m$-simplexe standard $\sigma_{E}$ du bord de $E$, une $C W$-équivalence

$$
|E| \stackrel{\sim}{\longrightarrow}|\Delta[n+1]|
$$

qui identifie $\left|\left(\sigma_{E}\right)\right|$ à $|(\sigma)|$ (ceci à l'aide de $n+1-m$ extensions coniques dont l'existence est un corollaire du théorème de Newman, §1.5). Une application itérée du lemme de Barratt montre donc que le domaine «comprimé » $\operatorname{Dom}(p) /\left(R_{\partial p}^{(1)}\right)$ est un $(n+1)$-élément simplicial.

Le domaine $\partial$-réduit $\overline{\operatorname{Dom}}(p)$ s'obtient à partir du domaine comprimé en identifiant successivement certains des $n$-simplexes du bord. Ces $n$-simplexes peuvent être supposés adjacents au moment de l'identification à condition d'introduire les relations $\left(\sigma_{1} \sim \sigma_{2}\right) \in R_{\partial p}^{(2)}$ selon le schéma de réduction sous-jacent à $\mathcal{A}_{\hat{\partial}_{j} p \rightarrow\left(\hat{\partial}_{j} p\right)_{\text {red }}}$. Les simplexes $\sigma_{1}, \sigma_{2}$ sont éventuellement modifiés sur leur bord par des compressions et/ou identifications introduites précédemment; un argument de récurrence montre cependant qu'ils engendrent des $n$-éléments simpliciaux qui s'intersectent selon un $(n-1)$-élément. Or, le quotient $E / E_{1} \sim E_{2}$ d'un $(n+1)$-élément par l'identification de deux $n$-éléments $E_{1}, E_{2} \subset b E$ s'intersectant selon un $(n-1)$-élément forme encore un $(n+1)$-élément, (cf. [R-S, 3.6]). Cela montre par récurrence que le domaine $\partial$-réduit est un $(n+1)$-élément simplicial.

Le morphisme $\varepsilon_{j}: \operatorname{Dom}\left(\partial_{j} p\right) \longrightarrow \overline{\operatorname{Dom}}(p)$ est déduit du morphisme composé

$$
\operatorname{Dom}\left(\hat{\partial}_{j} p\right) \stackrel{\widehat{\varepsilon_{j}}}{\longrightarrow} \operatorname{Dom}(p) \longrightarrow \overline{\operatorname{Dom}}(p)
$$

TOME $123-1995-\mathrm{N}^{\circ} 1$ 
par restriction convenable. Les relations $R_{\hat{\partial}_{j} p}^{(2)}$ créent en fait les adjacences nécessaires à la définition de $\varepsilon_{j}$. Les simplexes identifiés en un ne font plus partie du bord, puisque désormais face d'exactement deux $(n+1)$ simplexes. Cela montre que les faces latérales $\varepsilon_{j}\left(\operatorname{Dom}\left(\partial_{j} p\right)\right)$ jointes aux faces extrémales $e(\Delta[n]), s(\Delta[n])$ recouvrent le bord entier du domaine $\partial$-réduit.

\subsection{L'homotopie prismatique est 《ไtopologique 〉.}

Désignons par $|X|^{[0,1]}$ l'espace des chemins du réalisé $|X|$ : c'est à homotopie près, un groupoïde pour les fonctions structurelles :

$$
\begin{array}{r}
e_{|X|}, s_{|X|}:|X|^{[0,1]} \longrightarrow|X|: \gamma \mapsto \gamma(0), \gamma(1) \\
i_{|X|}:|X| \longrightarrow|X|^{[0,1]}: x \mapsto(\gamma(u) \equiv x)
\end{array}
$$

Nous allons préciser 1.3.c en montrant l'existence d'une application de graphes topologiques $\varphi:|I X| \rightarrow|X|^{[0,1]}$ qui préserve à homotopie près la structure groupoïdale de $|I X|$, ce qui implique en particulier :

$$
\left|e_{X}\right|=e_{|X|} \circ \varphi, \quad\left|s_{X}\right|=s_{|X|} \circ \varphi, \quad i_{|X|}=\varphi \circ\left|i_{X}\right|
$$

On procède en deux étapes : on définira d'abord un plongement de graphes naturel (compatible avec les structures multiplicatives)

$$
\widehat{\varphi}:|\widehat{I} X| \longleftrightarrow|X|^{[0,1]}
$$

Ensuite, il sera possible de montrer l'existence d'un couple $\left(\Phi_{t}, \varphi\right)$ tel que $\Phi_{t}$ soit une déformation graphique de $\widehat{\varphi}$ vers $\varphi \circ|q|$ :

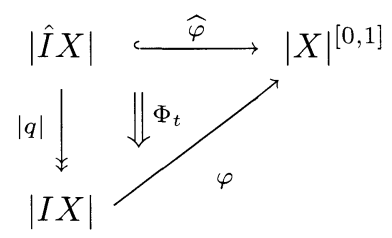

1.8.b. Le plongement graphique $\widehat{\varphi}$.

Rappelons que l'espace $|X|^{[0,-]}$ des chemins de Moore de $|X|$ est le sous-espace de $|X|^{\mathbb{R}^{+}} \times \mathbb{R}^{+}$formé par les couples $\left(\gamma_{\infty}, r\right)$ tels que le chemin

$$
\gamma_{\infty}: \mathbb{R}^{+} \longrightarrow|X|: u \mapsto \gamma_{\infty}(u)
$$


soit stationnaire pour $u \geq r$. Pour alléger la notation, on remplacera $\left(\gamma_{\infty}, r\right)$ par le couple $(\gamma, r)$ tel que $\gamma=\gamma_{\infty} \mid[0, r]$. L'inverse formel d'un chemin de Moore est défini par $(\gamma, r)^{-1}=\left(\gamma^{-1}, r\right)$; la composition (associative!) de chemins de Moore est définie par concaténation

$$
\left(\gamma_{1}, r_{1}\right)\left(\gamma_{2}, r_{2}\right) \cdots\left(\gamma_{\ell}, r_{\ell}\right)=\left(\gamma_{1} \cdots \gamma_{\ell}, r_{1}+\cdots+r_{\ell}\right)
$$

A tout $\widehat{\Gamma}_{n}(X)$-trajet $p=\left[\xi_{1}, i_{1}\right]^{\varepsilon_{1}} \ldots\left[\xi_{\ell}, i_{\ell}\right]^{\varepsilon_{\ell}} \in(\widehat{I} X)_{n}$ est alors associé un $n$-simplexe singulier $p_{\text {sing }}: \Delta_{n} \rightarrow|X|^{[0,-]}$ à valeurs dans l'espace des chemins de Moore de $|X|$. On pose en effet

$$
\begin{aligned}
p_{\text {sing }}: \Delta_{n} & \left.\longrightarrow X\right|^{[0,-]} \\
\left(t_{0} \ldots t_{n}\right) & \longmapsto\left[\xi_{1}, i_{1}\right]_{\text {sing }}^{\varepsilon_{1}}\left(t_{0} \ldots t_{n}\right) \cdots\left[\xi_{\ell}, i_{\ell}\right]_{\text {sing }}^{\varepsilon_{\ell}}\left(t_{0} \ldots t_{n}\right),
\end{aligned}
$$

où les chemins de Moore élémentaires sont définis par

$$
\begin{aligned}
{[\xi, i]_{\operatorname{sing}}\left(t_{0} \ldots t_{n}\right):\left[0, t_{i}\right] } & \longrightarrow|X| \\
u & \longmapsto|\xi|\left(t_{0} \ldots t_{i-1}, t_{i}-u, u, t_{i+1} \ldots t_{n}\right) .
\end{aligned}
$$

L'intervalle de définition du chemin de Moore composé $p_{\text {sing }}\left(t_{0} \ldots t_{n}\right)$ est en particulier égal à $\left[0, \sum_{k=1}^{\ell} t_{i_{k}}\right]$. L'associativité de la composition de chemins de Moore implique que la structure simpliciale du simplexe singulier $p_{\text {sing }}$ est compatible avec la structure simpliciale du $\widehat{\Gamma}_{n}(X)$ trajet $p$. De manière précise, on aura pour tout $j$ tel que $0 \leq j \leq n$,

$$
\left(\hat{\partial}_{j} p\right)_{\text {sing }}=\partial_{j} p_{\text {sing }}, \quad\left(\hat{s}_{j} p\right)_{\text {sing }}=s_{j} p_{\text {sing }} .
$$

Il suffit en effet d'établir ces formules de commutation pour les trajets élémentaires. A titre d'exemple nous montrons «la moins évidente»:

$$
\begin{aligned}
& \left(\hat{s}_{i}[\xi, i]\right)_{\text {sing }}\left(t_{0} \ldots t_{n+1}\right)(u) \\
& =\left(\left[s_{i} \xi, i+1\right]\left[s_{i+1} \xi, i\right]\right)_{\text {sing }}\left(t_{0} \ldots t_{n+1}\right)(u) \\
& =\left\{\begin{array}{l}
\left|s_{i} \xi\right|\left(t_{0} \ldots t_{i-1}, t_{i}, t_{i+1}-u, u, t_{i+2} \ldots t_{n+1}\right) \\
\left|s_{i+1} \xi\right|\left(t_{0} \ldots t_{i-1}, t_{i}-\left(u-t_{i+1}\right), u-t_{i+1}, t_{i+1} \ldots t_{n+1}\right)
\end{array}\right. \\
& =|\xi|\left(t_{0} \ldots t_{i-1}, t_{i}+t_{i+1}-u, u, t_{i+2} \ldots t_{n+1}\right) \\
& =s_{i}\left([\xi, i]_{\text {sing }}\right)\left(t_{0} \ldots t_{n+1}\right)(u) \text {. }
\end{aligned}
$$

Il s'ensuit qu'il existe une et une seule application continue

$$
\widehat{\varphi}_{M}:|\widehat{I} X| \longrightarrow|X|^{[0,-]}
$$

TOME $123-1995-\mathrm{N}^{\circ} 1$ 
telle que $\widehat{\varphi}_{M} \circ|p|=p_{\text {sing }}$ pour tout $p \in \widehat{I} X$, (cf. [Cu, 1.30]). Par construction même, $\widehat{\varphi}_{M}$ est injective et compatible avec les structures de graphes et les structures multiplicatives.

Le plongement graphique $\widehat{\varphi}:|\widehat{I} X| \hookrightarrow|X|^{[0,1]}$ est enfin le composé de $\widehat{\varphi}_{M}$ avec la normalisation $|X|^{[0,-]} \rightarrow|X|^{[0,1]}$ :

$$
\begin{aligned}
(\gamma, r) \longmapsto\|(\gamma, r)\|:[0,1] & \rightarrow|X| \\
u & \mapsto \gamma(r u) .
\end{aligned}
$$

1.8.c. La déformation graphique $\Phi_{t}$.

L'existence de $\Phi_{t}$ est liée au fait que les fibres ponctuelles de $|q|$ : $|\widehat{I} X| \rightarrow|I X|$ se contractent dans $|X|^{[0,1]}$, mais nous n'avons pas trouvé d'arguments généraux qui permettent de conclure. Aussi allons-nous donner une construction récursive de $\Phi_{t}$ en grimpant sur le squelette du $C W$-complexe $|\widehat{I} X|$.

Remarquons d'abord que $\Phi_{t}$ s'écrit localement sous forme d'applications

$$
\Phi_{t}^{p}: \Delta_{n} \times[0,1] \longrightarrow|X|^{[0,1]}
$$

ou encore par adjonction sous forme d'applications

$$
\Phi_{t, u}^{p}: \Delta_{n} \times[0,1]^{2} \longrightarrow|X|,
$$

$p \in(\widehat{I} X)_{n}, n \geq 0$. Pour définir une déformation graphique $\Phi_{t}$ de $\Phi_{0}=\widehat{\varphi}$ vers $\Phi_{1}=\varphi \circ|q|$, il faut et il suffit que les déformations locales $\Phi_{t, u}^{p}$ satisfassent aux conditions suivantes :

$(\mathrm{L} 1)_{p} \Phi_{0, u}^{p}: \Delta_{n} \times[0,1] \rightarrow|X|$ est l'adjoint de $\left\|p_{\text {sing }}\right\|: \Delta_{n} \longrightarrow|X|^{[0,1]} ;$

$(\mathrm{L} 2)_{p}$ si $p_{\text {red }}=p_{\text {red }}^{\prime}$, alors $\Phi_{1, u}^{p}=\Phi_{1, u}^{p^{\prime}}$;

$(\mathrm{L} 3)_{p} \Phi_{t, 0}^{p}$ et $\Phi_{t, 1}^{p}$ sont constantes en $t$;

$(\mathrm{L} 4)_{p} \Phi_{t, u}^{\hat{\partial}_{j} p}=\partial_{j} \Phi_{t, u}^{p}$ et $\Phi_{t, u}^{\hat{s}_{j} p}=s_{j} \Phi_{t, u}^{p}, 0 \leq j \leq \operatorname{dim} p$.

Nous ajoutons une condition supplémentaire qui exprime plus précisément de quelle façon les déformations locales seront faites :

$(\mathrm{L} 5)_{p}$ La déformation $\Phi_{t, u}^{p}$ se factorise par le domaine arborescent $\widetilde{\operatorname{Dom}}(p)$ :

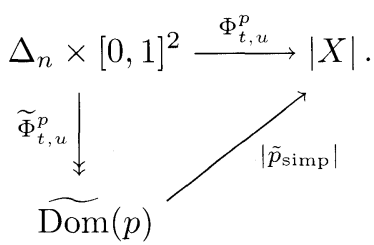

BULLETIN DE LA SOCIÉTÉ MATHÉMATIQUE DE FRANCE 
Le domaine arborescent du $\widehat{\Gamma}_{n}(X)$-trajet non trivial $p \in(\widehat{I} X)_{n}$ est défini (en reprenant les notations de 1.6.b) comme le quotient

$$
\widetilde{\operatorname{Dom}}(p)=\operatorname{Dom}(p) /\left(R_{p}^{(1)} \cup \widetilde{R}_{p}^{(2)}\right)
$$

où l'ensemble de relations $\widetilde{R}_{p}^{(2)}$ contient une relation $\alpha_{k_{1}} \sim \alpha_{k_{2}}$ pour tout aller retour $\left[\xi_{k_{1}}, i_{k_{1}}\right]^{\varepsilon_{k_{1}}}\left[\xi_{k_{2}}, i_{k_{2}}\right]^{\varepsilon_{k_{2}}}$ tel que le trajet entouré

$$
\left[\xi_{k_{1}+1}, i_{k_{1}+1}\right]^{\varepsilon_{k_{1}+1}} \cdots\left[\xi_{k_{2}-1}, i_{k_{2}-1}\right]^{\varepsilon_{k_{2}-1}}
$$

se réduise au trajet trivial. Il y a en général une inclusion stricte $R_{p}^{(2)} \varsubsetneqq \widetilde{R}_{p}^{(2)}$, mais $\widetilde{R}_{p}^{(2)}$ a l'avantage d'être indépendant du schéma de réduction.

Le point-clé de la construction récursive de $\Phi_{t}$ est l'observation suivante (comparer avec $[\mathrm{M}, 3.6])$ :

1.8.d. LeMme. - Le domaine arborescent d'un $\widehat{\Gamma}_{n}(X)$-trajet $p \in(\widehat{I} X)_{n}$ se rétracte par déformation topologique sur le domaine du n-prisme associé $p_{\text {red }} \in(I X)_{n}$ de sorte que $\tilde{p}_{\text {simp } \mid \operatorname{Dom}\left(p_{\text {red }}\right)}=\left(p_{\text {red }}\right)_{\text {simp }}$. Le domaine arborescent est en particulier contractile pour tout trajet $p \in \widehat{I} X$.

A titre d'exemple, soit $p$ un $\widehat{\Gamma}_{0}(X)$-trajet. Supposons en plus que le $\widehat{\Gamma}_{0}(X)$-trajet comprimé s'écrive formellement

$$
a b b^{-1} c c^{-1} c d e^{-1} e f f^{-1} d^{-1} g .
$$

Le domaine arborescent de $p$ et le domaine du $n$-prisme associé $p_{\text {red }}$ se présentent alors comme suit :
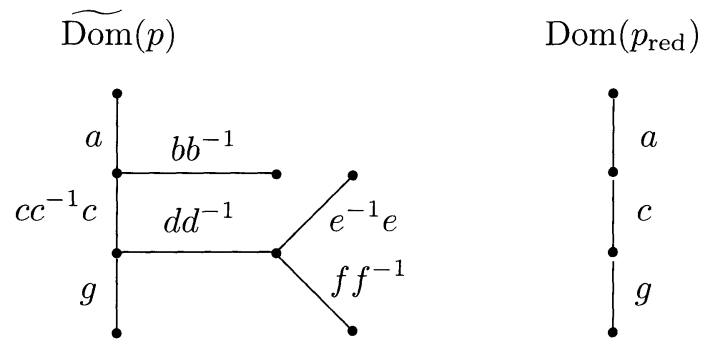

Il n'est pas difficile de montrer qu'en toute généralité, le domaine arborescent d'un $\Gamma_{0}(X)$-trajet est un arbre contenant le domaine du 0-prisme associé comme tronc (i.e. sous-arbre linéaire), d'où la déformation de rétraction cherchée. Au tronc s'attachent en particulier des branches qui sont structurées selon la configuration des aller retour.

TOME $123-1995-\mathrm{N}^{\circ} 1$ 
Le cas d'un $\Gamma_{n}(X)$-trajet $p$ est traité de façon analogue, les arêtes étant remplacées par des $(n+1)$-simplexes munis d'un indice directionnel. L'écriture formelle du trajet $p$ permet en effet de construire comme ci-dessus un arbre $\mathcal{A}_{p}$. La déformation de rétraction de $\mathcal{A}_{p}$ sur $\mathcal{A}_{p_{\text {red }}}$ induit une déformation de rétraction de $|\widetilde{\operatorname{Dom}}(p)| \operatorname{sur}\left|\operatorname{Dom}\left(p_{\text {red }}\right)\right|$, la paire $\left(|\widetilde{\operatorname{Dom}}(p)|,\left|\operatorname{Dom}\left(p_{\text {red }}\right)\right|\right)$ étant paramétrée par la paire

$$
\left(\Delta_{n} \times \mathcal{A}_{p}, \Delta_{n} \times \mathcal{A}_{p_{\text {red }}}\right)
$$

à l'aide du diagramme commutatif suivant (dans lequel $\alpha$ désigne le $n$ prisme fondamental de $\operatorname{Dom}(p))$ :

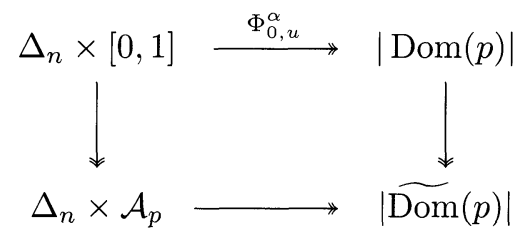

La paramétrisation $\Delta_{n} \times \mathcal{A}_{p} \longrightarrow|\widetilde{\operatorname{Dom}}(p)|$ n'est en général bijective (et ouverte) qu'en restriction à l'intérieur du $n$-simplexe affine $\Delta_{n}$, mais cela suffit.

Revenons à la condition locale $(\text { L5 })_{p}$ : il nous faudra de plus une condition de cohérence des factorisations $\widetilde{\Phi}_{t, u}^{p}: \Delta_{n} \times[0,1]^{2} \rightarrow \widetilde{\operatorname{Dom}}(p)$ par rapport aux opérateurs de face telle que la condition (L4) en soit une conséquence. La définition de l'ensemble de relations $\widetilde{R}_{p}^{(2)}$ implique que pour tout indice $j$, tel que $0 \leq j \leq \operatorname{dim} p$, l'image du morphisme composé

$$
\operatorname{Dom}\left(\hat{\partial}_{j} p\right) \stackrel{\widehat{\varepsilon_{j}}}{\longrightarrow} \operatorname{Dom}(p) \longrightarrow \widetilde{\operatorname{Dom}}(p),
$$

notée $\partial_{j} \widetilde{\operatorname{Dom}}(p)$, se projette canoniquement sur le domaine arborescent $\widetilde{\operatorname{Dom}}\left(\hat{\partial}_{j} p\right)$, projection qu'on notera $q_{j}$. La condition de cohérence est alors la suivante :

$(\mathrm{L} 6)_{p}$ les restrictions $\partial_{j} \widetilde{\Phi}_{t, u}^{p}$ sont à valeurs dans $\left|\partial_{j} \widetilde{\operatorname{Dom}}(p)\right|$ et on a

$$
\left|q_{j}\right| \circ\left(\partial_{j} \widetilde{\Phi}_{t, u}^{p}\right)=\widetilde{\Phi}_{t, u}^{\hat{\partial}_{j} p}, \quad 0 \leq j \leq \operatorname{dim} p .
$$

La donnée d'un système de factorisations $\widetilde{\Phi}_{t, u}^{\hat{\partial}_{j} p}$ vérifiant $(\mathrm{L} 6)_{\hat{\partial}_{j} p}$ pour $0 \leq j \leq \operatorname{dim} p$, induit une factorisation

$$
\widetilde{\Phi}_{t, u}^{\hat{\partial} p}: b \Delta_{n} \times[0,1]^{2} \longrightarrow \widetilde{\operatorname{Dom}}(p) ;
$$

autrement dit, les conditions de cohérence (L6) permettent de «recoller ». 
La construction récursive de $\Phi_{t}$ procède alors comme suit. On suppose $\Phi_{t}$ définie sur le $(n-1)$-squelette de $|\widehat{I} X|$ de sorte que les conditions locales (L1)-(L6) soient vérifiées, et on l'étend au $n$-squelette par la construction suivante (l'argument vaudra pour $n=0$ ) : pour tout $\widehat{\Gamma}_{n}(X)$-trajet (non dégénéré) réduit $p_{\text {red }}$, les conditions (L1), (L3), (L4) déterminent et définissent $\Phi_{t, u}^{p_{\text {red }}}$ sur le sous-espace

$$
U_{n}=b \Delta_{n} \times[0,1]^{2} \cup \Delta_{n} \times\{(t, u) ; t=0 \text { ou } u=0 \text { ou } u=1\}
$$

de $\Delta_{n} \times[0,1]^{2}$. Les conditions $(\mathrm{L} 5)_{\hat{\partial} p_{\text {red }}}$ et $(\mathrm{L} 6)_{\hat{\partial} p_{\text {red }}}$ jointes à l'isomorphisme $\operatorname{Dom}\left(p_{\text {red }}\right) \cong \widetilde{\operatorname{Dom}}\left(p_{\text {red }}\right)$ permettent de factoriser cette application par le domaine arborescent. La factorisation s'étend à $\Delta_{n} \times[0,1]^{2}$ selon une déformation qui rétracte $\Delta_{n} \times[0,1]^{2}$ sur $U_{n}$ (c'est le principe de l'extension homotopique).

Ensuite, pour un $\widehat{\Gamma}_{n}(X)$-trajet (non dégénéré) non réduit $p$, les conditions (L1) $)_{p}-(\mathrm{L} 4)_{p}$ déterminent et définissent la déformation locale $\Phi_{t, u}^{p}$ sur le bord entier

$$
b\left(\Delta_{n} \times[0,1]^{2}\right)=b \Delta_{n} \times[0,1]^{2} \cup \Delta_{n} \times b[0,1]^{2}
$$

de $\Delta_{n} \times[0,1]^{2}$. Les conditions (L5) $)_{\hat{\partial} p}$ et (L6) $\hat{\partial}_{p}$ jointes au Lemme 1.8.d permettent de factoriser cette application par le domaine arborescent. Puisque celui-ci est contractile, la factorisation s'étend à $\Delta_{n} \times[0,1]^{2}$. L'extension satisfait en particulier à $(\mathrm{L} 6)_{p}$. Cela termine la construction récursive de $\Phi_{t}$ et en même temps la démonstration de la PropoSITION 1.3.c.

\section{Le fibré en lacets}

2.1. - Le groupoïde simplicial $I X$ contient pour tout ensemble simplicial pointé et connexe $(X, *)$ un modèle du fibré en lacets qu'on définit par $\Omega X=\left(e_{X}, s_{X}\right)^{-1}(*, *), P X=\left(e_{X}, s_{X}\right)^{-1}(X, *)$ et $q=e_{X \mid P X}: P X \rightarrow X$. La projection $q$ est a priori une $\Omega X$-fibration principale (i.e. $\Omega X$ opère sans isotropie sur $P X$ de sorte que les orbites de l'action s'identifient aux fibres de $q$ ), mais la théorie générale des fibrations simpliciales montre que toute fibration principale est localement triviale (cf. 3.1), ce qui justifie notre terminologie. Pour tout $n \geq 0$, le groupe $(\Omega X)_{n}$ est libre en tant que groupe d'automorphismes du point base $*_{n} \in X_{n}$ à l'intérieur du groupoïde libre $(I X)_{n}$. Nous expliciterons au $\S 3.2$, dans le cas d'un ensemble simplicial réduit $X$, des bases canoniques pour les groupes $(\Omega X)_{n}$ ainsi qu'une structure canonique de $\Omega X$-fibré principal sur $P X$.

TOME $123-1995-\mathrm{N}^{\circ} 1$ 
2.2. - L'objectif de cette partie est d'étudier les isomorphismes de connexion

$$
\partial_{n+1}(X): \pi_{n+1}(|X|) \stackrel{\sim}{\longrightarrow} \pi_{n}(|\Omega X|)
$$

de la suite exacte longue d'homotopie du fibré en lacets. Comme notre intérêt est de nature combinatoire, il convient de définir les groupes d'homotopie en question en passant par la réalisation géométrique et non pas par plongement dans une enveloppe de Kan (cf. [G-Z], [K1]).

Les groupes d'homotopie du groupe simplicial $\Omega X$ admettent toutefois une définition directe à l'aide du complexe de Moore $\left((\widetilde{\Omega X})_{n}, \widetilde{\partial}_{n}\right)_{n \geq 0}$; nos conventions sont les suivantes :

$$
\begin{aligned}
(\widetilde{\Omega X})_{n} & =(\Omega X)_{n} \cap \operatorname{ker} \partial_{0} \cap \operatorname{ker} \partial_{1} \cap \cdots \cap \operatorname{ker} \partial_{n-1}, \\
\widetilde{\partial}_{n} & =\partial_{n \mid(\widetilde{\Omega X})_{n}}, \\
\pi_{n}(\Omega X) & =\operatorname{ker} \widetilde{\partial}_{n} / \operatorname{im} \widetilde{\partial}_{n+1} .
\end{aligned}
$$

Par la théorie de Moore-Kan [K1], on sait en effet que la réalisation géométrique induit des isomorphismes $\pi_{n}(\Omega X) \cong \pi_{n}(|\Omega X|)$ pour $n \geq 0$, associant aux $n$-cycles $\omega \in \operatorname{ker} \widetilde{\partial}_{n}$ du complexe de Moore les représentants simpliciaux $|\underline{\omega}|:\left|S^{n}\right| \rightarrow|\Omega X|$, où

$$
\underline{\omega}=\omega / b \Delta[n]: \Delta[n] / b \Delta[n] \longrightarrow \Omega X .
$$

Or, l'existence d'une contraction prismatique de l'espace total du fibré en lacets nous permettra $(\S \S 2.5-2.6)$ d'associer à tout $n$-cycle $\omega$ du complexe de Moore de $\Omega X$ un représentant simplicial canonique de la classe d'homotopie adjointe

$$
\partial_{n+1}(X)^{-1}([|\underline{\omega}|]) \in \pi_{n+1}(|X|) .
$$

Le représentant adjoint, noté $\omega_{\mathrm{sph}}: \Sigma^{n+1}(\omega) \rightarrow X$, se déduit directement de la structure prismatique du $n$-cycle $\omega$. Pour motiver la définition de $\omega_{\mathrm{sph}}$, nous introduisons le concept de «piste» (track) d'un prisme, concept que nous avons emprunté à BARRATt [Ba].

2.3. Définition. - La piste $[p]$ d'un prisme $p \in I X$ est sa classe d'homotopie fibre rel. le bord par rapport à la fibration $\left(e_{X}, s_{X}\right): I X \rightarrow$ $X \times X$.

Comme les fibres $(I X)_{x, y}$ vérifient la condition de Kan, deux $n$-prismes $p_{1}, p_{2} \in(I X)_{x, y}$ appartiennent à la même piste si et seulement s'il existe 
un $(n+1)$-prisme $R \in(I X)_{x, y}$ tel que $[R, n]: p_{1} \sim p_{2}$ soit une homotopie élémentaire rel. le bord (remarque 1.4).

Nous noterons $\operatorname{Tr}_{x, y}(X)$ l'ensemble des pistes d'entrée $x$ et de sortie $y$. La composition de prismes induit une structure de groupe sur $\operatorname{Tr}_{x, x}(X)$. On notera $\operatorname{Tr}_{x}(X)$ le sous-groupe de $\operatorname{Tr}_{x, x}(X)$ formé par les pistes-lacets qui sont triviales sur leur bord. Il résulte alors des définitions qu'on a pour le point base $*$ de $X$ des isomorphismes canoniques

$$
\operatorname{Tr}_{*_{n}}(X) \cong \pi_{n}(\Omega X), \quad n \geq 0
$$

Les représentants sont en effet les mêmes et la différence de deux $n$-cycles $\omega_{1}, \omega_{2} \in(\Omega X)_{n}$ est un bord du complexe de Moore si et seulement si les deux cycles appartiennent à la même piste.

\subsection{Le représentant simplicial adjoint.}

Rappelons qu'en topologie le groupe des pistes-lacets au-dessus de l'application constante $\left|S^{n}\right| \stackrel{*}{\rightarrow}|X|$ sert de description commune aux groupes $\pi_{n+1}(|X|)$ et $\pi_{n}(\Omega|X|)$ réalisant ainsi l'adjonction entre les foncteurs «suspension» et «espace de lacets» $[\mathrm{Ba}]$.

Par analogie, nous définissons le diagramme commutatif suivant :

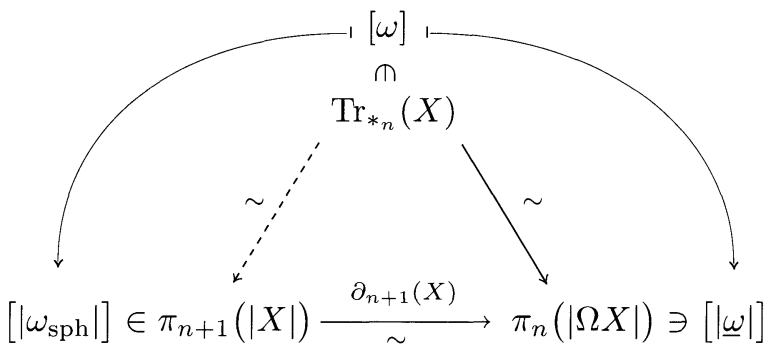

Compte tenu de la construction topologique, il est naturel de définir le représentant simplicial adjoint $\omega_{\mathrm{sph}}: \Sigma^{n+1}(\omega) \rightarrow X$ en quotientant «par le bord » le morphisme $\bar{\omega}_{\text {simp }}: \overline{\operatorname{Dom}}(\omega) \rightarrow X$ associé au $n$-cycle $\omega(\S 1.6)$ :

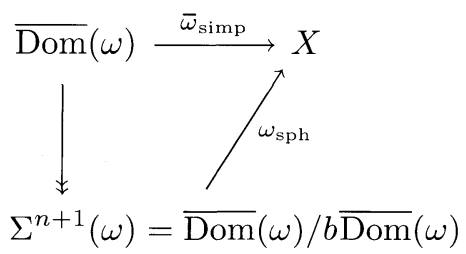

En effet, le bord du $(n+1)$-élément simplicial $\overline{\operatorname{Dom}}(\omega)$ est réunion du bord latéral et du bord extrémal (§1.7.b). Comme les faces du $n$-cycle $\omega$ TOME $123-1995-\mathrm{N}^{\circ} 1$ 
sont triviales, le bord latéral est vide ( $\S 1.7 . \mathrm{a})$; comme $\omega$ est un «lacet», le bord extrémal est appliqué sur le point base de $X$, ce qui montre que la définition de $\omega_{\mathrm{sph}}$ est consistante. La structure simpliciale de la $(n+1)$ sphère $\Sigma^{n+1}(\omega)$ dépend uniquement de la structure formelle du $n$-cycle $\omega$ et des schémas de réductions $\mathcal{A}_{\hat{\partial}_{j} \omega \rightarrow\left(\hat{\partial}_{j} \omega\right)_{\text {red }}}$ de ses faces.

Il reste à montrer que la classe d'homotopie $\left[\left|\omega_{\mathrm{sph}}\right|\right] \in \pi_{n+1}(|X|)$ vérifie effectivement que $\partial_{n+1}(X)\left(\left[\left|\omega_{\text {sph }}\right|\right]\right)=[|\underline{\omega}|]$. Nous proposons à cet effet une démonstration entièrement combinatoire qui utilise la contractibilité prismatique de l'espace total du fibré en lacets.

2.5. Lemme. - Le groupoïde simplicial IX est muni d'une déformation prismatique $\rho_{X}: I X \rightarrow I(I X): \mathrm{id}_{I X} \sim i_{X} \circ s_{X}$ qui rétracte $I X$ sur $X$ en fixant $X$. La déformation vérifie en outre

$$
I e_{X} \circ \rho_{X}=\operatorname{id}_{I X} \quad \text { et } I s_{X} \circ \rho_{X}=i_{X} \circ s_{X} \text {. }
$$

En particulier, pour un ensemble simplicial pointé et connexe $(X, *)$, l'espace total du fibré en lacets admet la contraction prismatique $\rho_{X \mid P X}$ : $P X \rightarrow P(P X)$.

Preuve. - Le morphisme $\rho_{X}$ est défini par récurrence sur la longueur des prismes en posant pour tout simplexe $x \in X, \rho_{X}\left(1_{x}\right)=1_{\left(1_{x}\right)}$, et pour tout prisme composé $[\xi, i]^{\varepsilon} \cdot p \in I X$,

$$
\rho_{X}\left([\xi, i]^{\varepsilon} \cdot p\right)=\left[\mu[\xi, i]^{\varepsilon} \cdot s_{i} p, i\right]^{\varepsilon} \cdot \rho_{X}(p),
$$

où

$$
\mu[\xi, i]^{\varepsilon}= \begin{cases}{\left[s_{i+1} \xi, i\right]} & \text { si } \varepsilon=1, \\ {\left[s_{i} \xi, i+1\right]^{-1}} & \text { si } \varepsilon=-1\end{cases}
$$

Plusieurs vérifications sont à faire : on observe d'abord que le prisme $\mu[\xi, i]^{\varepsilon}$ forme la composante droite du prisme dégénéré $s_{i}[\xi, i]^{\varepsilon}$; en particulier $s_{X}\left(\mu[\xi, i]^{\varepsilon}\right)=e_{X}\left(s_{i} p\right)$. On vérifie ensuite la formule

$$
\left(e_{I X}, s_{I X}\right)\left(\left[\mu[\xi, i]^{\varepsilon}, i\right]^{\varepsilon}\right)=\left([\xi, i]^{\varepsilon}, 1_{s_{X}\left([\xi, i]^{\varepsilon}\right)}\right),
$$

d'où les identités

$$
e_{I X} \circ \rho_{X}=\mathrm{id}_{I X} \quad \text { et } \quad s_{I X} \circ \rho_{X}=i_{X} \circ s_{X}=I s_{X} \circ \rho_{X} \text {. }
$$

Par récurrence sur la longueur des prismes, il s'ensuit d'une part que

$$
s_{I X}\left(\left[\mu[\xi, i]^{\varepsilon} \cdot s_{i} p, i\right]^{\varepsilon}\right)=e_{I X}\left(\rho_{X}(p)\right),
$$

i.e. la définition de $\rho_{X}$ est consistante, et d'autre part que $I e_{X} \circ \rho_{X}=\mathrm{id}{ }_{I X}$. 
Enfin, pour montrer que $\rho_{X}$ commute avec les opérateurs simpliciaux, il suffit - par un argument de récurrence — de vérifier les formules suivantes :

$$
\begin{array}{r}
\partial_{j}\left[\mu[\xi, i]^{\varepsilon}, i\right]^{\varepsilon}= \begin{cases}{\left[\mu \partial_{j}[\xi, i]^{\varepsilon}, i-1\right]^{\varepsilon}} & \text { si } j<i, \\
1_{s_{X}\left([\xi, i]^{\varepsilon}\right)} & \text { si } j=i, \\
{\left[\mu \partial_{j}[\xi, i]^{\varepsilon}, i\right]^{\varepsilon}} & \text { si } j>i ;\end{cases} \\
s_{j}\left[\mu[\xi, i]^{\varepsilon}, i\right]^{\varepsilon}= \begin{cases}{\left[\mu s_{j}[\xi, i]^{\varepsilon}, i+1\right]^{\varepsilon}} & \text { si } j<i, \\
\rho_{X}\left(s_{i}[\xi, i]^{\varepsilon}\right) & \text { si } j=i, \\
{\left[\mu s_{j}[\xi, i]^{\varepsilon}, i\right]^{\varepsilon}} & \text { si } j>i .\end{cases}
\end{array}
$$

2.6. Proposition. - Soit $(X, *)$ un ensemble simplicial pointé et connexe. Tout n-cycle $\omega$ du complexe de Moore de $\Omega X$ s'inscrit alors dans le diagramme commutatif suivant:

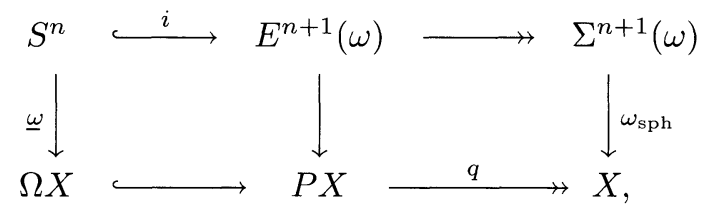

dans lequel $E^{n+1}(\omega)$ désigne un $(n+1)$-élément simplicial tel que

$$
i S^{n}=b E^{n+1}(\omega) \quad \text { et } \quad \Sigma^{n+1}(\omega)=E^{n+1}(\omega) / b E^{n+1}(\omega) .
$$

La longueur de la chaîne qui constitue le cycle fondamental de la $(n+1)$ sphère $\Sigma^{n+1}(\omega)$ est en particulier égale à la longueur du n-cycle $\omega$.

Preuve. - Il suffit d'expliciter $E^{n+1}(\omega)$ et de compléter le diagramme. Le $n$-cycle $\omega \in(\Omega X)_{n} \subset(P X)_{n}$ se contracte dans $P X$ selon le $n$-prisme $\Omega=\rho_{X}(\omega) \in(P(P X))_{n}$ qui est d'entrée $\omega$, de sortie $1_{*_{n}}$ et de bord trivial. Le morphisme associé $\Omega_{\text {simp }}: \overline{\operatorname{Dom}}(\Omega) \rightarrow P X$ se factorise donc par la face-sortie, définissant ainsi le morphisme vertical médian ci-dessus $\left(E^{n+1}(\omega)=\overline{\operatorname{Dom}}(\Omega) / s(\Delta[n])\right)$ :

$$
\bar{\Omega}_{\text {simp }} / s(\Delta[n]): E^{n+1}(\omega) \longrightarrow P X .
$$

En effet, par application itérée du lemme de Barratt (cf. 1.7), $E^{n+1}(\omega)$ est un $(n+1)$-élément simplicial de bord $e(\Delta[n]) / e(b \Delta[n])$. L'inclusion canonique $i: S^{n} \cong b E^{n+1}(\omega) \hookrightarrow E^{n+1}(\omega)$ rend commutatif le carré gauche ci-dessus. Le quotient $E^{n+1}(\omega) / b E^{n+1}(\omega)$ s'identifie à $\Sigma^{n+1}(\omega)$ (§ 2.4) en vertu de l'isomorphisme canonique $\overline{\operatorname{Dom}}(\Omega) \cong \overline{\operatorname{Dom}}(\omega)$ qui résulte de l'identité $P q \circ\left(\rho_{X \mid P X}\right)=\operatorname{id}_{P X}(\S 2.5)$. 
Par construction même de la $(n+1)$-sphère simpliciale $\Sigma^{n+1}(\omega)$, les $(n+1)$-simplexes qui constituent son cycle fondamental sont en bijection avec les composantes élémentaires du $n$-cycle $\omega$.

2.7. Remarque.

(a) Le théorème d'approximation semi-simpliciale [F-P, 4.6.25] fournit l'existence d'une $C W$-subdivision finie $\widetilde{\Sigma}^{n+1}(\omega)$ de $S^{n+1}$ et d'un morphisme $\widetilde{\omega}_{\text {sph }}: \widetilde{\Sigma}^{n+1}(\omega) \rightarrow X$ réalisant simplicialement la classe d'homotopie adjointe de $[\underline{\omega}] \in \pi_{n}(\Omega X) \cong \pi_{n}(|\Omega X|) \cong \pi_{n}(\Omega|X|)$. Par ailleurs, nous verrons au $\S 3.3$ que la construction $\Omega X$ n'est d'un point de vue algébrique guère plus qu'une extension un peu mystérieuse du groupe de lacets $G X$ de Kan.

La nouveauté ici est le lien explicite qui s'est établi entre :

- la représentation «simpliciale» $\omega_{\mathrm{sph}}: \Sigma^{n+1}(\omega) \rightarrow X$ et

- la représentation «algébrique» $\underline{\omega}: S^{n} \rightarrow \Omega X$.

On pourra étudier à ce sujet l'exemple donné au $\S 3.4$.

(b) Nous terminons cette partie en montrant que le groupoïde des composantes connexes de $I X$ par rapport à l'homotopie fibre 2.3 s'identifie canoniquement au groupoïde fondamental $\Pi X$ tel qu'il a été défini par GABRIEL-Zisman [G-Z, II.7.1]. Notre définition ressemble cependant plus à la définition topologique $[\mathrm{Br}]$. On déduit en particulier l'équivalence entre la définition «standard» du groupe de Poincaré d'un ensemble simplicial pointé $(X, *)$ et la nôtre (via $\pi_{0}(\Omega X)$ ). La Proposition 2.6 est élémentaire dans cette dimension.

Le groupoïde fondamental $\Pi X$ est défini dans [G-Z] comme le quotient du groupoïde libre $(I X)_{0}$ par l'ensemble générateur des relations

$$
R_{x}:\left[\partial_{1} x, 0\right]_{\mathrm{red}} \sim\left(\left[\partial_{2} x, 0\right]\left[\partial_{0} x, 0\right]\right)_{\mathrm{red}}, \quad x \in X_{2} .
$$

Il s'agit de montrer que :

(i) pour tout $x \in X_{2}$, les 0 -prismes $\left[\partial_{1} x, 0\right]_{\text {red }}$ et $\left(\left[\partial_{2} x, 0\right]\left[\partial_{0} x, 0\right]\right)_{\text {red }}$ appartiennent à la même piste;

(ii) deux 0 -prismes quelconques $p_{1}, p_{2}$ appartenant à la même piste sont reliés par une suite de relations du type $1_{\rho_{1}} R_{x}^{\varepsilon} 1_{\rho_{2}}$, avec $x \in X_{2}$ et $\rho_{1,2} \in(I X)_{0}, \varepsilon= \pm 1$.

$\operatorname{Ad}(i)$ : pour $R=\left[s_{0} \partial_{2} x, 1\right][x, 1]\left[s_{1} \partial_{1} x, 0\right]$ on vérifie que $[R, 0]$ est l'homotopie élémentaire cherchée (comparer avec $3.5 \mathrm{~d}$ ).

$\operatorname{Ad}(i i):$ si $R=\left[\xi_{1}, i_{1}\right]^{\varepsilon_{1}} \cdots\left[\xi_{\ell}, i_{\ell}\right]^{\varepsilon_{\ell}} \in(I X)_{1}$ est tel que $[R, 0]: p_{1} \sim p_{2}$, alors l'homotopie élémentaire $[R, 0]$ est réalisable par une suite de relations $1_{\rho_{1}^{k}} R_{\xi_{k}}^{\varepsilon_{k}} 1_{\rho_{2}^{k}}, 1 \leq k \leq \ell$, pour des 0 -prismes $\rho_{1,2}^{k}$ convenablement choisis. 


\section{Le fibré universel de Kan et la construction cobar d'Adams-Baues}

\subsection{Le fibré universel de Kan.}

La théorie des fonctions de torsion (twisting functions) offre un formalisme commode pour traiter les fibrés simpliciaux. En effet, la donnée conjointe d'une $G$-fibration principale

$$
G \longleftrightarrow E \stackrel{q}{\longrightarrow} X
$$

(cf. 2.1) et d'une pseudosection

$$
j: X \rightarrow E: x \mapsto j_{x}
$$

(i.e. $q \circ j=\operatorname{id}_{X}, s_{i} j_{x}=j_{s_{i} x}, 0 \leq i \leq \operatorname{dim} x, \partial_{i} j_{x}=j_{\partial_{i} x}, 0 \leq i<\operatorname{dim} x$, mais en général $\left.\partial_{n} j_{x} \neq j_{\partial_{n} x}, n=\operatorname{dim} x\right)$ définit une et une seule fonction de torsion (de degré -1$) t: X \rightarrow G$ vérifiant $\partial_{n} j_{x}=\left(j_{\partial_{n} x}\right)^{t_{n} x}, x \in X_{n}$, $n>0$. L'intérêt de cette fonction de torsion provient du $G$-isomorphisme

$$
\varphi: X \times_{t} G \longrightarrow E:(x, g) \longmapsto\left(j_{x}\right)^{g}
$$

qui munit $E$ d'une structure de produit cartésien tordu (twisted cartesian product). Or, un produit cartésien tordu est localement trivial, i.e. $E$ est un $G$-fibré principal (cf. $[\mathrm{Cu}, 6.8]$ ).

Si l'ensemble simplicial $X$ est réduit (i.e. $X_{0}=\left\{*_{0}\right\}$ ), il admet une fonction de torsion universelle

$$
t^{G}: X \longrightarrow G X
$$

de sorte que pour toute fonction de torsion $t: X \rightarrow G$, il existe un et un seul homomorphisme de groupes simpliciaux $\varphi: G X \rightarrow G$ tel que $\varphi \circ t^{G}=t$. La construction d'une fonction de torsion universelle est due à KAN [K1]. Celui-ci montre en particulier que le produit cartésien tordu $E X=X \times_{t^{G}} G X$ est topologiquement contractile, ce qui lui fournit une définition algébrique des groupes d'homotopie de $|X|$ basée sur la famille des isomorphismes de connexion $\pi_{n+1}(|X|) \stackrel{\sim}{\longrightarrow} \pi_{n}(G X)$ pour $n \geq 0$.

Nous précisons le résultat de Kan du côté géométrique en montrant que le fibré universel de Kan est - en vertu de sa propriété universelle plongé dans le fibré en lacets $\Omega X \hookrightarrow P X \rightarrow X$ de manière invariante par rapport à la contraction prismatique du dernier. Le plongement dépend d'une structure de produit cartésien tordu sur $P X$ resp. d'une pseudosection de la projection $q: P X \rightarrow X$ que nous explicitons cidessous. 


\subsection{Un arbre maximal du graphe $\Gamma_{n}(X)$.}

Soit $(X, *)$ un ensemble simplicial réduit. Pour tout $n>0$, on définit un arbre maximal du graphe $\Gamma_{n}(X)=\left(X_{n}, \Xi_{n}, e, s\right)(\S 1.2)$ en posant :

$T_{n}=\left\{[\xi, i] \in \Xi_{n} ; i \neq n, \xi\right.$ dégénéré dans les directions $\left.i+1, i+2, \ldots, n\right\}$.

On montre en effet que tout $n$-simplexe $x \in X_{n}$ est joint au point base $*_{n} \in X_{n}$ selon un unique $\left(X_{n}, T_{n}, e, s\right)$-trajet réduit qu'on notera $j_{x}$. Le trajet est explicitement donné par la formule

$j_{x}=\left(\left[s_{n} x, n-1\right]\left[s_{n} s_{n-1} \partial_{n-1} x, n-2\right] \cdots\left[s_{n} s_{n-1} \cdots s_{1} \partial_{1} \cdots \partial_{n-1} x, 0\right]\right)_{\mathrm{red}}$.

L'unicité d'un $\left(X_{n}, T_{n}, e, s\right)$-trajet réduit d'entrée $x$ et de sortie $*_{n}$ se démontre par récurrence sur la «distance» du $n$-simplexe $x$ au point base $*_{n}$, formellement définie comme le plus petit entier $i$ tel que $x$ s'écrive sous la forme $x=s_{n-1} \cdots s_{i+1} s_{i} y$ avec $y \in X_{i}$. Nous laissons les détails au lecteur. La description explicite de l'arbre maximal $\left(X_{n}, T_{n}, e, s\right)$ permet de définir une base $B_{n}$ du groupe libre $(\Omega X)_{n}$ (cf. 2.1). Il résulte en effet des définitions que l'ensemble

$$
B_{n}=\left\{\left(j_{\partial_{i+1} \xi}\right)^{-1}[\xi, i] j_{\partial_{i} \xi} ;[\xi, i] \in\left(\Xi_{n} \backslash T_{n}\right)\right\}
$$

forme une base de $(\Omega X)_{n}$.

3.3. Proposition. - Soit $(X, *)$ un ensemble simplicial réduit.

(a) La fonction $j: X \rightarrow P X: x \mapsto j_{x}$ est une pseudosection de $q: P X \rightarrow X$. La fonction de torsion associée s'écrit

$$
t_{n+1}^{\Omega}: X_{n+1} \longrightarrow(\Omega X)_{n}: \xi \longmapsto\left(j_{\partial_{n+1} \xi}\right)^{-1}[\xi, n] j_{\partial_{n} \xi} .
$$

(b) Le groupe de lacets universel $G X$ est isomorphe au sous-groupe simplicial de $\Omega X$ engendré par l'image de la fonction de torsion. Le groupe $(G X)_{n}$ s'identifie en particulier au sous-groupe de $(\Omega X)_{n}$ librement engendré par les n-prismes $\bar{\xi}=t_{n+1}^{\Omega} \xi$, où $\xi \in\left(X_{n+1} \backslash s_{n} X_{n}\right)$.

(c) La contraction prismatique $\rho_{X \mid P X}: P X \rightarrow P(P X)$ induit par restriction une contraction prismatique $\rho_{X \mid E X}: E X \rightarrow P(E X)$. En particulier, comme les groupes simpliciaux $G X$ et $\Omega X$ sont libres (au sens de Kan), ils ont même type d'homotopie multiplicative.

Preuve.

(a) On vérifie aisément que les contractions prismatiques

$$
j_{x}: x \sim s_{n-1} \cdots s_{0} \partial_{0} \cdots \partial_{n-1} x
$$

BULLETIN DE LA SOCIÉTÉ MATHÉMATIQUE DE FRANCE 
commutent avec les opérateurs simpliciaux exceptés $\partial_{n}$, avec $n=\operatorname{dim} x$. D'après 3.1 , on a :

$$
t_{n+1}^{\Omega} \xi=\left(j_{\partial_{n+1} \xi}\right)^{-1}\left(\partial_{n+1} j_{\xi}\right)=\left(j_{\partial_{n+1} \xi}\right)^{-1}[\xi, n] j_{\partial_{n} \xi} .
$$

(b) Il existe un unique homomorphisme de groupes simpliciaux

$$
\varphi: G X \longrightarrow \Omega X
$$

tel que $\varphi(\bar{\xi})=t^{\Omega}(\xi)$ (cf. 3.1). Les $n$-prismes $t^{\Omega}(\xi), \xi \in\left(X_{n+1} \backslash s_{n} X_{n}\right)$ font partie de la base $B_{n}$ de $(\Omega X)_{n}$ ce qui prouve l'injectivité de $\varphi$.

(c) L'inclusion $\varphi: G X \hookrightarrow \Omega X$ induit une inclusion des fibrés principaux associés $E X \hookrightarrow P X$, les $n$-simplexes du fibré universel s'identifiant aux $n$-prismes $p \in(P X)_{n}$ dont les composantes élémentaires $[\xi, i]^{\varepsilon}$ vérifient soit $i=n$ soit $[\xi, i] \in T_{n}$. L'opérateur $\mu$ du $\S 2.5$ préserve cette condition ce qui implique que la contraction prismatique de $P X$ se restreint à $E X$.

En vertu du lemme des cinq, l'inclusion $\varphi$ est alors une équivalence d'homotopie faible. Or d'après un théorème de Kan [K2], une équivalence d'homotopie faible entre groupes simpliciaux libres est une équivalence d'homotopie multiplicative (rappelons qu'un groupe simplicial $G$ est libre, si pour tout $n \geq 0$, le groupe $G_{n}$ est librement engendré par un ensemble $B_{n}$ en sorte que la réunion des $B_{n}$ soit stable par opérateurs de dégénérescence). Dans notre cas, la réunion des bases $B_{n}$ de $(\Omega X)_{n}$ n'est stable par opérateurs de dégénérescence qu'en restriction au sous-groupe simplicial $G X$. Pour appliquer le théorème de Kan, nous allons modifier les bases $B_{n}$ par des transformations de Tietze itérées en sorte que les nouvelles bases $B_{n}^{\prime}$ soient stables et qu'en plus $B_{n}^{\prime} \cap G X=B_{n} \cap G X$.

Pour cela, nous associons à tout $(n+1)$-simplexe $\xi \in X_{n+1}$ et tout indice $i, 0 \leq i \leq n$, un entier positif ou nul $\operatorname{def}_{i} \xi$, maximal pour la propriété que $\xi$ soit dégénéré dans les directions $i+1, i+2, \ldots, i+\operatorname{def}_{i} \xi$. On pose :

$$
\begin{aligned}
& \llbracket \xi, i \rrbracket= \begin{cases}j_{\partial_{i+1} \xi}[\xi, i] j_{\partial_{i} \xi} & \operatorname{sidef}_{i} \xi=0, \\
s_{i+r-1} \cdots s_{i+1} s_{i}\left(j_{\partial_{i+1} \eta}^{-1}[\eta, i] j_{\partial_{i} \eta}\right) & \text { si } \operatorname{def}_{i} \xi=r>0\end{cases} \\
& \xi=s_{i+r} \cdots s_{i+2} s_{i+1} \eta .
\end{aligned}
$$

Les ensembles

$$
B_{n}^{\prime}=\left\{\llbracket \xi, i \rrbracket ;[\xi, i] \in\left(\Xi_{n} \backslash T_{n}\right)\right\}, \quad n \geq 0,
$$

TOME $123-1995-\mathrm{N}^{\circ} 1$ 
vérifient alors :

(i) $B_{n}^{\prime}$ est une base de $(\Omega X)_{n}$,

(ii) $s_{i} B_{n}^{\prime} \subset B_{n+1}^{\prime}$ pour $0 \leq i \leq n$,

(iii) $B_{n}^{\prime} \cap G X=B_{n} \cap G X$.

L'assertion (i) résulte d'une récurrence sur les entiers $\operatorname{def}_{i} \xi$. On pose en effet

$$
\begin{aligned}
B_{n}^{(r)}=\{\llbracket \xi, i \rrbracket ;[\xi, i] & \left.\notin T_{n}, \operatorname{def}_{i} \xi \leq r\right\} \\
& \cup\left\{j_{\partial_{i+1} \xi}^{-1}[\xi, i] j_{\partial_{i} \xi} ;[\xi, i] \notin T_{n}, \operatorname{def}_{i} \xi>r\right\} ;
\end{aligned}
$$

en particulier :

$$
B_{n}^{(0)}=B_{n} \quad \text { et } \quad B_{n}^{(n-1)}=B_{n}^{\prime} .
$$

Par hypothèse de récurrence on peut alors supposer que $B_{n}^{(r)}$ est une base de $(\Omega X)_{n}$. Pour tout $n$-prisme élémentaire $[\xi, i] \notin T_{n}$ tel que $\operatorname{def}_{i} \xi=r+1$ et $\xi=s_{i+r+1} \cdots s_{i+2} s_{i+1} \eta$, on obtient :

$$
\llbracket \xi, i \rrbracket=s_{i+r} \cdots s_{i}\left(j_{\partial_{i+1} \eta}^{-1}[\eta, i] j_{\partial_{i} \eta}\right)=\prod_{i+r+1 \geq k \geq i}\left(j_{\partial_{k+1} \xi_{k}}^{-1}\left[\xi_{k}, k\right] j_{\partial_{k} \xi_{k}}\right),
$$

où

$$
\xi_{k}= \begin{cases}\xi & \text { si } k=i, \\ s_{k-1} \cdots s_{i} \partial_{i+1} \cdots \partial_{k} \xi & \text { si } i+r+1 \geq k>i .\end{cases}
$$

Comme $\operatorname{def}_{k} \xi_{k} \leq r$ pour $i+r+1 \geq k>i$, le $n$-prisme $\llbracket \xi, i \rrbracket$ s'écrit

$$
\llbracket \xi, i \rrbracket=\left(\prod \llbracket \widetilde{\xi}_{k}, k \rrbracket\right) j_{\partial_{i+1} \xi}^{-1}[\xi, i] j_{\partial_{i} \xi}
$$

avec $\operatorname{def}_{k} \widetilde{\xi}_{k} \leq r$. On peut donc effectuer la modification $B_{n}^{(r)} \mapsto B_{n}^{(r+1)}$ par des transformations de Tietze qui substituent les éléments $\llbracket \xi, i \rrbracket \in$ $\left(B_{n}^{(r+1)} \backslash B_{n}^{(r)}\right)$ aux éléments $j_{\partial_{i+1} \xi}^{-1}[\xi, i] j_{\partial_{i} \xi}$.

Les assertions (ii) et (iii) sont immédiates si l'on écrit les bases $B_{n}^{\prime}$ sous la forme équivalente

$$
B_{n}^{\prime}=\left\{s_{i+r-1} \cdots s_{i} \llbracket \eta, i \rrbracket ; \operatorname{def}_{i} \eta=0,[\eta, i] \in\left(\Xi_{n-r} \backslash T_{n-r}\right)\right\} .
$$

\subsection{Exemple : un représentant simplicial fini de la fibration de Hopf $\left|S^{\mathbf{3}}\right| \rightarrow\left|S^{\mathbf{2}}\right|$.}

La fibration de Hopf de la 3-sphère sur la 2-sphère est à homotopie et orientation près caractérisée par la propriété d'être un «générateur» 
du groupe $\pi_{3}\left(\left|S^{2}\right|\right)$. Selon 2.5 et 3.3.c, on a un triangle commutatif d'isomorphismes :

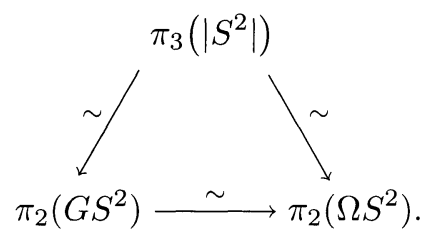

KAN [K1] a explicité un représentant de la classe génératrice de $\pi_{2}\left(G S^{2}\right)$, à savoir le commutateur simple

$$
\omega=s_{0} \bar{\sigma} \cdot s_{1} \bar{\sigma} \cdot s_{0} \bar{\sigma}^{-1} \cdot s_{1} \bar{\sigma}^{-1} \in\left(G S^{2}\right)_{2},
$$

où $\sigma$ désigne le simplexe fondamental de $S^{2}$. L'écriture prismatique 3.3.a-3.3.b de ce commutateur est :

$$
\omega=\left[s_{0} \sigma, 2\right]\left[s_{1} \sigma, 2\right]\left[s_{2} \sigma, 1\right]\left[s_{0} \sigma, 2\right]^{-1}\left[s_{2} \sigma, 1\right]^{-1}\left[s_{1} \sigma, 2\right]^{-1} \in\left(\Omega S^{2}\right)_{2} .
$$

Selon 2.6, on obtient donc un représentant simplicial $\omega_{\text {sph }}: \Sigma^{3}(\omega) \rightarrow S^{2}$ de la fibration de Hopf tel que le cycle fondamental de la 3-sphère soit constitué de six 3-simplexes.

En reprenant les notations de 1.6, la 3-sphère s'écrit comme quotient

$$
\Sigma^{3}(\omega)=\overline{\operatorname{Dom}}(\omega) / b \overline{\operatorname{Dom}}(\omega),
$$

où

$$
\overline{\operatorname{Dom}}(\omega)=\operatorname{Dom}(\omega) /\left(R_{\partial \omega}^{(1)} \cup R_{\partial \omega}^{(2)}\right)
$$

Si l'on note

$$
\left[\alpha_{1}, 2\right]\left[\alpha_{2}, 2\right]\left[\alpha_{3}, 1\right]\left[\alpha_{4}, 2\right]^{-1}\left[\alpha_{5}, 1\right]^{-1}\left[\alpha_{6}, 2\right]^{-1}
$$

le 2-prisme fondamental de $\operatorname{Dom}(\omega)$, alors l'ensemble de relations $R_{\partial \omega}^{(1)} \cup$ $R_{\partial \omega}^{(2)}$ s'écrit en codimension 1 :

$$
\begin{array}{r}
\left\{\left(\partial_{0} \alpha_{2} \sim s_{1} \partial_{1} \partial_{0} \alpha_{2}\right),\left(\partial_{0} \alpha_{3} \sim s_{0} \partial_{0} \partial_{0} \alpha_{3}\right),\left(\partial_{0} \alpha_{5} \sim s_{0} \partial_{0} \partial_{0} \alpha_{5}\right),\right. \\
\left.\left(\partial_{0} \alpha_{6} \sim s_{1} \partial_{1} \partial_{0} \alpha_{6}\right),\left(\partial_{0} \alpha_{1} \sim \partial_{0} \alpha_{4}\right)\right\} \\
\cup\left\{\left(\partial_{1} \alpha_{1} \sim \partial_{1} \alpha_{6}\right),\left(\partial_{1} \alpha_{2} \sim \partial_{1} \alpha_{4}\right)\right\} \\
\cup\left\{\left(\partial_{3} \alpha_{3} \sim \partial_{3} \alpha_{5}\right)\right\}
\end{array}
$$

(correspondant aux réductions des faces $\left.\hat{\partial}_{j} \omega, j=0,1,2\right)$. Le passage au quotient $\overline{\operatorname{Dom}}(\omega) \rightarrow \Sigma^{3}(\omega)$ se réalise par les relations $\partial_{3} \alpha_{1} \sim *_{2} \sim \partial_{3} \alpha_{6}$. Le représentant $\omega_{\mathrm{sph}}$ est enfin défini par $\alpha_{1}, \alpha_{4} \mapsto s_{0} \sigma, \alpha_{2}, \alpha_{6} \mapsto s_{1} \sigma$, $\alpha_{3}, \alpha_{5} \mapsto s_{2} \sigma$. 


\subsection{La construction cobar d'Adams-Baues.}

ADAms définit dans [A] la construction cobar $\Omega C$ d'une coalgèbre différentielle $C$. Il démontre que pour la coalgèbre différentielle $C_{*}(|X|)$ des chaînes d'un $C W$-complexe 1-réduit $|X|$, les complexes de chaînes $\Omega C_{*}(|X|)$ et $C_{*}(\Omega|X|)$ ont même type d'homotopie. Sa démonstration repose sur l'approximation par un $n$-cube de l'espace des chemins reliant premier et dernier sommet $\mathrm{du}(n+1)$-simplexe affine. Ces « approximations d'Adams » ont été formalisées par BAuEs [Bau] qui définit en particulier pour tout ensemble simplicial 1-réduit $X$ un $C W$-complexe «cubique» $\left\|\Omega_{B} X\right\|$ dont le complexe de chaînes $C_{*}\left(\left\|\Omega_{B} X\right\|\right)$ est isomorphe à la construction cobar d'Adams $\Omega C_{*}(|X|)$. Nous allons plonger le modèle cubique de Baues, subdivisé simplicialement, dans le monoïde simplicial $\Omega^{+} X$ des lacets prismatiques positifs. Le plongement revient à rendre «prismatiques» les approximations d'Adams.

Rappelons tout d'abord la définition de $\Omega_{B} X$ sous une forme qui nous sera commode (cf. [Bau, I.2.7-13]).

3.5.a. - BAuEs introduit la catégorie $\Omega \Delta$ : les objets de $\Omega \Delta$ sont les cordes (simpliciales)

$$
\mathrm{Cd}_{i_{1} i_{2} \cdots i_{\ell}}=\Delta\left[i_{1}+1\right] \square \Delta\left[i_{2}+1\right] \square \cdots \square \Delta\left[i_{\ell}+1\right]
$$

définies par amalgamation itérée (cf. 1.6) des diagrammes

$$
\Delta[0] \underset{s_{k}}{\stackrel{e_{k}}{\longrightarrow}} \Delta\left[i_{k}+1\right],, \quad 1 \leq k \leq \ell,
$$

$e_{k}$ (resp. $s_{k}$ ) désignant le sommet d'indice 0 (resp. $i_{k}+1$ ). Un nœud est un sommet de la corde égal à un des $e_{k}(\Delta[0])$ ou $s_{k}(\Delta[0]), 1 \leq k \leq \ell$. Tout sommet qui n'est pas un nœud est appelé interne. Les morphismes de $\Omega \Delta$ sont les morphismes simpliciaux qui préservent nœuds initial et final.

Le modèle cubique $\Omega_{B} X$, subdivisé simplicialement, est alors défini (X étant 1-réduit) comme le produit tensoriel $\Omega_{X} \underset{\Omega \Delta}{\otimes} L$ du foncteur contravariant

$$
\begin{aligned}
\Omega_{X}: \Omega \Delta & \longrightarrow \text { Ens (la catégorie des ensembles) } \\
\operatorname{Cd}_{i_{1} \cdots i_{\ell}} & \longmapsto \operatorname{Mor}_{\text {Esimp }}\left(\operatorname{Cd}_{i_{1} \cdots i_{\ell}}, X\right) \\
\varphi & \longmapsto \operatorname{Mor}_{\text {Esimp }}(\varphi, X)
\end{aligned}
$$

et du foncteur covariant

$$
\begin{aligned}
& L: \Omega \Delta \longrightarrow \text { Esimp (la catégorie des ensembles simpliciaux) } \\
& \mathrm{Cd}_{i_{1} \cdots i_{\ell}} \longmapsto \Delta[1]^{i_{1}+\cdots+i_{\ell}} \\
& \varphi \longmapsto L \varphi .
\end{aligned}
$$


La définition de $L \varphi$ découle de l'interprétation suivante du $\left(i_{1}+\cdots+i_{\ell}\right)$ cube simplicial $\Delta[1]^{i_{1}+\cdots+i_{\ell}}$, (cf. [Bau, III.3]) :

- L'ensemble $\mathcal{S}_{i_{1} \cdots i_{\ell}}^{\text {int }}$ des sommets internes de la corde $\operatorname{Cd}_{i_{1} \cdots i_{\ell}}$ est de cardinalité $i_{1}+\cdots+i_{\ell}$.

- L'ensemble $\mathcal{P}\left(\mathcal{S}_{i_{1} \cdots i_{\ell}}^{\text {int }}\right)$ des parties de $\mathcal{S}_{i_{1} \cdots i_{\ell}}^{\text {int }}$ est un ensemble partiellement ordonné dont le nerf $\Delta \mathcal{P}\left(\mathcal{S}_{i_{1} \cdots i_{\ell}}^{\text {int }}\right)$ s'identifie au $\left(i_{1}+\cdots+i_{\ell}\right)$-cube simplicial $\Delta[1]^{i_{1}+\cdots+i_{\ell}}$; en fait $\mathcal{P}\left(\mathcal{S}_{i_{1} \cdots i_{\ell}}^{\text {int }}\right) \cong[1]^{i_{1}+\cdots+i_{\ell}}$ et le nerf $\Delta$ commute avec le produit cartésien.

- Pour tout morphisme de cordes $\varphi: \mathrm{Cd}_{i_{1} \cdots i_{\ell}} \rightarrow \mathrm{Cd}_{j_{1} \cdots j_{m}}$, on note $N_{i_{1} \cdots i_{\ell}}$ l'ensemble des nœuds de $\mathrm{Cd}_{i_{1} \cdots i_{\ell}}$, et on définit

$$
\mathcal{P}(\varphi): \mathcal{P}\left(\mathcal{S}_{i_{1} \cdots i_{\ell}}^{\mathrm{int}}\right) \rightarrow \mathcal{P}\left(\mathcal{S}_{j_{1} \cdots j_{m}}^{\mathrm{int}}\right): \tau \mapsto\left(\varphi(\tau) \cup \varphi\left(N_{i_{1} \cdots i_{\ell}}\right)\right) \cap \mathcal{S}_{j_{1} \cdots j_{m}}^{\mathrm{int}}
$$

et $L \varphi=\Delta \mathcal{P}(\varphi)$.

Il est intéressant d'observer que le foncteur $L$ (essentiellement les approximations d'Adams) intervient également dans la théorie des (co)limites homotopiques de R.M. Vogt. Le lecteur pourra consulter l'article [Co] de Cordier, où ce lien est rendu explicite. A cet effet, il est utile de remarquer que la sous-catégorie de $\Omega \Delta$ formée par les morphismes de cordes élémentaires $\mathrm{Cd}_{n} \rightarrow \mathrm{Cd}_{m}$ est isomorphe à la catégorie simpliciale opposée $\Delta^{\mathrm{op}}$ (voir [Co, I.2]). La restriction de $L$ à cette sous-catégorie définit en particulier un foncteur contravariant $\Delta \rightarrow$ Esimp : $[n] \mapsto \Delta[1]^{n}$, noté $P$ dans $[\mathrm{Co}]$.

3.5.b. L'inclusion $\Omega_{B} X \hookrightarrow \Omega^{+} X$. - Pour tout ensemble simplicial $X$, $I^{+} X$ désigne le sous-ensemble simplicial de $I X$ formé par les prismes dont toutes les composantes sont d'exposant positif. Il existe alors pour toute corde $\mathrm{Cd}_{i_{1} \cdots i_{\ell}}$ une inclusion (cf. 3.5.c)

$$
k_{i_{1} \cdots i_{\ell}}: \Delta[1]^{i_{1}+\cdots+i_{\ell}} \longrightarrow\left(I^{+} \mathrm{Cd}_{i_{1} \cdots i_{\ell}}\right)_{e_{1}, s_{\ell}}
$$

induisant une transformation naturelle $k: L \dot{\rightarrow} I_{e, s}^{+}$de foncteurs $\Omega \Delta \rightarrow$ Esimp. La naturalité de $k$ implique que

$$
\begin{aligned}
i_{B}: \Omega_{X} \otimes L & \longrightarrow \Omega^{+} X \\
& \frac{\left(\sigma_{i_{1} \cdots i_{\ell}}, \gamma\right)}{} \longmapsto\left(I \sigma_{i_{1} \cdots i_{\ell}}\right)\left(k_{i_{1} \cdots i_{\ell}}(\gamma)\right)
\end{aligned}
$$

est bien défini.

L'injectivité de $i_{B}$ est une conséquence de l'injectivité de $k_{i_{1} \cdots i_{\ell}}$ et des deux faits suivants : 
(i) chaque classe $\overline{\left(\sigma_{i_{1} \cdots i_{\ell}}, \gamma\right)} \in \Omega_{X} \otimes_{\Omega \Delta} L$ admet un représentant unique $\left(\sigma_{i_{1} \cdots i_{\ell}}, \gamma\right) \in \Omega_{X} \times L$ tel que $\sigma_{i_{1} \cdots i_{\ell}}$ soit un $\ell$-uplet de simplexes non dégénérés de $X$ et tel que $\gamma$ soit un simplexe intérieur du cube simplicial $\Delta[1]^{i_{1}+\cdots+i_{\ell}}$ (cf. [Bau, II.1.5-8] et [F-P, 4.2.7]).

(ii) un simplexe intérieur de $\Delta[1]^{i_{1}+\cdots+i_{\ell}}$ est transformé par $k_{i_{1} \cdots i_{\ell}}$ en un prisme qui «passe» exactement une fois par chaque élément de la corde $\mathrm{Cd}_{i_{1} \cdots i_{\ell}}$ en suivant l'ordre.

3.5.c. Une version prismatique des approximations d'Adams. - Nous appelons régulier tout $n$-prisme positif $p \in\left(I^{+} X\right)_{n}$ dont les composantes $[\xi, i]$ vérifient soit $i=n$, soit $[\xi, i] \in T_{n}$ (i.e. le $(n+1)$-simplexe $\xi$ est dégénéré dans les directions $i+1, i+2, \ldots, n$; comparer avec 3.2-3.3). Le sous-ensemble simplicial de $I^{+} X$ formé par les prismes réguliers sera noté $I_{\text {reg }}^{+} X$. Comme il y a une inclusion naturelle $\left(I_{\text {reg }}^{+}\right)_{e, s} \stackrel{\bullet}{\hookrightarrow}\left(I^{+}\right)_{e, s}$, nous aurons terminé en montrant qu'il existe un isomorphisme naturel de foncteurs monö̈daux $\ell:\left(I_{\text {reg }}^{+}\right)_{e, s} \cong L: \Omega \Delta \rightarrow$ Esimp. A cet effet, il suffit d'expliciter la famille des isomorphismes $\ell_{n}:\left(I_{\text {reg }}^{+} \Delta[n+1]\right)_{e, s} \cong \Delta[1]^{n}$, pour $n \geq 0$; les composantes $\ell_{i_{1} \cdots i_{\ell}}$ s'en déduisent par amalgamation, puisque les foncteurs $\left(I_{\text {reg }}^{+}\right)_{e, s}$ et $L$ sont monoïdaux.

Pour ce faire, remarquons d'abord que la fonction entrée-sortie $(m \geq 0)$

$$
\left(e_{\Delta[n+1]}, s_{\Delta[n+1]}\right): \Xi_{m} \longrightarrow \Delta[n+1]_{m} \times \Delta[n+1]_{m}
$$

est injective d'image l'ensemble des «sauts» de l'ordre partiel

$$
\Delta[n+1]_{m}=\operatorname{Mor}_{\Delta}([m],[n+1]),
$$

où nous entendons par saut tout couple de $m$-simplexes

$$
\sigma_{0}, \sigma_{1}:[m] \rightarrow[n+1]
$$

tels que $\sigma_{0} \leq \sigma_{1}$ (i.e. $\sigma_{0}(i) \leq \sigma_{1}(i), 0 \leq i \leq m$ ), mais $\sigma_{0}(i) \neq \sigma_{1}(i)$ pour exactement un indice $i$. L'indice «directionnel 》 du saut correspond en fait à l'indice directionnel de l'unique $m$-prisme élémentaire $[\xi, i]$ d'entrée $\sigma_{0}$ et de sortie $\sigma_{1}$. Nous dirons que le saut $\left(\sigma_{0}, \sigma_{1}\right)$ est régulier si le $m$-prisme élémentaire associé l'est, ce qui veut simplement dire qu'on a

$$
\sigma_{1}(i)=\sigma_{1}(i+1)=\cdots=\sigma_{1}(m) .
$$

Un $m$-prisme régulier $p \in\left(I_{\text {reg }}^{+} \Delta[n+1]\right)_{m}$ est donc formellement équivalent à une suite $\left(\sigma_{k}\right)_{0 \leq k \leq \ell}$ de $m$-simplexes $\sigma_{k} \in \Delta[n+1]_{m}$ telle que les couples $\left(\sigma_{k-1}, \sigma_{k}\right)$ de simplexes consécutifs soient des sauts réguliers. Les faces $\partial_{j} p$ (resp. les dégénérescences $s_{j} p$ ) correspondent aux suites $\left(\partial_{j} \sigma_{k}\right)_{0 \leq k \leq \ell}\left(\operatorname{resp} .\left(s_{j} \sigma_{k}\right)_{0 \leq k \leq \ell}\right)$ étant entendu que les éventuelles répétitions dans la suite $\left(\partial_{j} \sigma_{k}\right)_{0 \leq k \leq \ell}$ sont éliminées (resp. que les éventuels «doubles sauts》 dans la suite $\left(s_{j} \sigma_{k}\right)_{0 \leq k \leq \ell}$ sont subdivisés en deux «simples sauts 》). 
L'isomorphisme cherché admet alors la forme suivante (cf. 3.5.a) :

$$
\begin{aligned}
\ell_{n}:\left(I_{\mathrm{reg}}^{+} \Delta[n+1]\right)_{e, s} \stackrel{\sim}{\longrightarrow}[1]^{n}=\Delta \mathcal{P}\left(\mathcal{S}_{n}^{\mathrm{int}}\right) \\
\quad \hat{\equiv}\left(\sigma_{k}\right)_{0 \leq k \leq \ell} \longmapsto\left(\tau_{0} \subseteq \tau_{1} \subseteq \cdots \subseteq \tau_{\operatorname{dim} p}\right),
\end{aligned}
$$

où l'ensemble de sommets $\tau_{j} \in \mathcal{P}\left(\mathcal{S}_{n}^{\text {int }}\right), 0 \leq j \leq \operatorname{dim} p$, est défini par la formule

$$
\tau_{j}=\left(\bigcup_{0 \leq k \leq \ell} \sigma_{k}(j)\right) \backslash\{0, n+1\} .
$$

La régularité de $p$ implique que la définition est consistante. L'application $\ell_{n}$ commute avec les opérateurs simpliciaux. La fonctorialité de $\ell$ par rapport aux morphismes de cordes élémentaires $\mathrm{Cd}_{n} \rightarrow \mathrm{Cd}_{m}$ est immédiate. La fonctorialité par rapport aux «subdivisions de cordes》 $\mathrm{Cd}_{i_{1} \cdots i_{\ell}} \longleftrightarrow \mathrm{Cd}_{n}$ résulte de la définition de $L$ et du fait que l'intersection

$$
\bigcap_{0 \leq j \leq \operatorname{dim} p} \tau_{j}
$$

s'identifie à l'ensemble des sommets $(\neq 0, n+1)$ de la suite $p \widehat{\equiv}\left(\sigma_{k}\right)_{0 \leq k \leq \ell}$. Puisque les morphismes élémentaires et les subdivisions de cordes engendrent par composition et amalgamation tous les morphismes de $\Omega \Delta$, la fonctorialité de $\ell$ est ainsi établie.

Pour montrer que l'application $\ell_{n}$ est bijective nous explicitons l'application inverse $k_{n}:$ soit $\left(\tau_{0} \subseteq \cdots \subseteq \tau_{m}\right) \in\left(\Delta \mathcal{P}\left(\mathcal{S}_{n}^{\text {int }}\right)\right)_{m}$; le $m$-prisme régulier

$$
k_{n}\left(\left(\tau_{0} \subseteq \cdots \subseteq \tau_{m}\right)\right) \in\left(I_{\text {reg }}^{+} \Delta[n+1]\right)_{m}
$$

est alors défini par l'unique suite de $m$-simplexes $\left(\sigma_{k}\right)_{0 \leq k \leq \ell}$ qui vérifie

(i) $\sigma_{0} \equiv 0, \sigma_{\ell} \equiv n+1$, et

(ii) le couple $\left(\sigma_{k-1}, \sigma_{k}\right)$, pour $1 \leq k \leq \ell$, forme le saut régulier d'indice directionnel minimal $i$ tel qu'on ait l'inclusion

$$
\left\{\sigma_{k-1}(i), \sigma_{k}(i)\right\} \subseteq \tau_{i} \cup\{0, n+1\}
$$

3.5.d. Illustration (cf. [Bau, p. 102]). — Les simplexes $\sigma:[m] \rightarrow[n+1]$ sont désignés par la suite de leurs valeurs.

TOME $123-1995-\mathrm{N}^{\circ} 1$ 

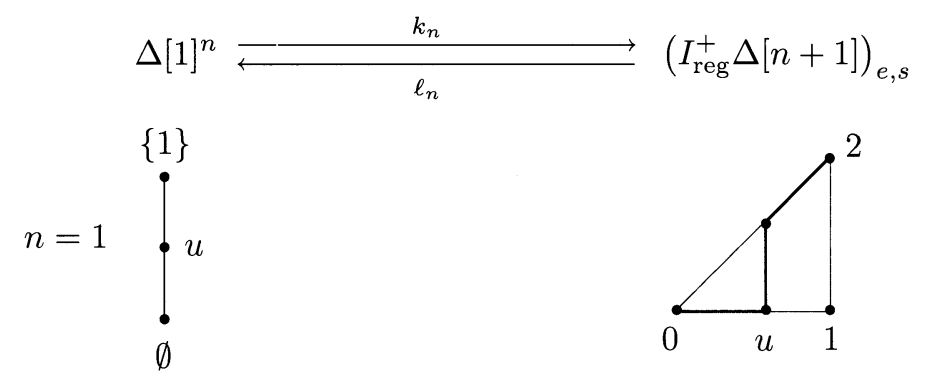

$$
(\emptyset \subset\{1\}) \longleftrightarrow \begin{aligned}
& (00,01,02,22) \hat{=} \\
& {[001,1][012,1][022,0]}
\end{aligned}
$$
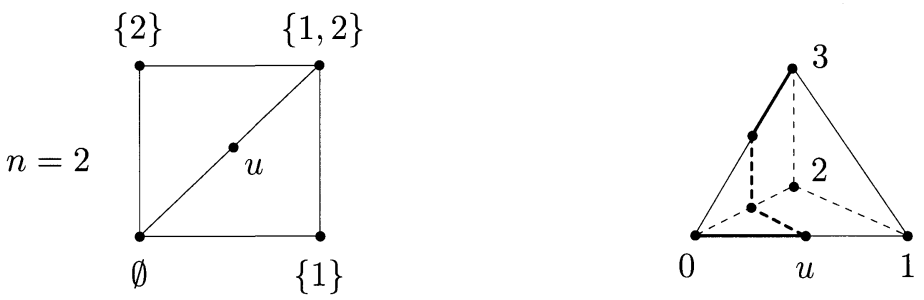

$$
\begin{aligned}
& (\emptyset \subset\{1,2\}) \longleftrightarrow \begin{array}{l}
(00,01,02,03,33) \hat{=} \\
{[001,1][012,1][023,1][033,0]}
\end{array} \\
& (\emptyset \subset\{1\} \subset\{1,2\}) \longleftrightarrow \begin{array}{l}
(000,001,011,012,013,033,333) \widehat{=} \\
{[0001,2][0011,1][0112,2][0123,2][0133,1][0333,0]}
\end{array} \\
& (\emptyset \subset\{2\} \subset\{1,2\}) \longleftrightarrow \begin{array}{l}
(000,001,002,022,023,033,333) \widehat{=} \\
{[0001,2][0012,2][0022,1][0223,2][0233,1][0333,0]}
\end{array}
\end{aligned}
$$

\section{BIBLIOGRAPHIE}

[A] Adams (J.-F.). - On the cobar construction, Proc. Nat. Acad. Sci. USA, t. 42, 1956, p. 409-412.

[Ba] Barratt (M.-G.). - Track groups, Proc. London Math. Soc., t. 5, 3, 1955, p. 71-106. 
[Bau] Baues (H.-J.). - Geometry of loop spaces and the cobar construction. - Mem. Amer. Math. Soc. 230, 1980.

[Be] Berger (C.). - Un modèle simplicial fibrant de l'espace des chemins, C.R. Acad. Sci. Paris, série I, t. 315, 1992, p. 193-196.

[Br] Brown (R.). - Topology : a geometric account of general topology, homotopy types and the fundamental groupoid . - Ellis Horwood series, New York, 1988.

[Co] Cordier (J.-M.). - Sur les limites homotopiques de diagrammes homotopiquement cohérents, Compositio Math., t. 62, 1987, p. 367-388.

[Cu] Curtis (E.-B.). - Simplicial homotopy theory, Adv. in Math., t. 6, 1971, p. 107-209.

[D] Duskin (J.). - Free groupoids, trees and free groups, J. Pure and Appl. Algebra, t. 68, 1990, p. 95-108.

[F-P] Fritsch (R.) and Piccinini (R.A.).-Cellular structures in topology, Cambridge Studies in Adv. Math., t. 19, 1990.

[G-Z] Gabriel (P.) and Zisman (M.). - Calculus of fractions and homotopy theory. - Ergebnisse der Math., Bd. 35, Springer Verlag, 1967.

[K1] Kan (D.M.). - A combinatorial definition of homotopy groups, Ann. of Math., t. 67, 1958, p. 282-312.

[K2] Kan (D.M.). - On homotopy theory and c.s.s. groups, Ann. of Math., t. 68,1958, p. $38-53$.

[M] Milnor (J.). - Construction of universal bundles I, Ann. of Math., t. 63, 1956, p. 272-284.

[Q] Quillen (D.G.). - Homotopical algebra, Lecture Notes in Math. 43, Springer Verlag, 1967 .

[R-S] Rourke (C.P.) and Sanderson (B.J.). - Introduction to Piecewiselinear topology. - Ergebnisse der Math., Bd. 69, Springer Verlag, 1972 .

TOME $123-1995-\mathrm{N}^{\circ} 1$ 